Rahul Kumar*, Achchhe Lal, B. N. Singh, and Jeeoot Singh

\title{
Meshfree approach on buckling and free vibration analysis of porous FGM plate with proposed IHHSDT resting on the foundation
}

https://doi.org/10.1515/cls-2019-0017

Received Jun 30, 2019; accepted Aug 16, 2019

\begin{abstract}
In the present work, new inverse hyperbolic higher-order shear deformation theory (IHHSDT) is proposed and implemented for buckling analysis and free vibration analysis of porous Functionally Graded Material (FGM) plate on the foundation. The proposed theory follows the approximately parabolic distribution of the transverse stresses through the plate thickness and satisfies the conditions of continuity and differentiability. Three different types of porosity distribution considered. Governing differential equations (GDEs) of the plate is developed in the framework of proposed theories by Hamilton's principle. Multiquadrics radial basis function (MQ-RBF) based Meshfree method used for discretizing the GDEs. The result obtained by the present theory is validated with the three-dimensional elastic theory and other available solutions in the literature to ensure the efficacy and accuracy of the proposed theory. Numerical results obtained for buckling and free vibration for porous FGM plate resting on the foundation. Effect of grading index, porosity fraction, porosity distribution, the effect of foundation, and the span to thickness ratio have discussed. The secured results can consider as a benchmark for future studies.
\end{abstract}

Keywords: Meshfree; Buckling; Free Vibration; IHHSDT; Porosity

\footnotetext{
*Corresponding Author: Rahul Kumar: Department of Mechanical Engineering, SVNIT Surat 395007 India; Email: rahul22mech@gmail.com

Achchhe Lal: Department of Mechanical Engineering, SVNIT Surat 395007 India

B. N. Singh: Department of Aerospace Engineering, Indian Institute of Technology Kharagpur, West Bengal 721302

Jeeoot Singh: Department of Mechanical Engineering, Madan Mohan Malaviya University of Technology, Gorakhpur, U.P, 273010, India
}

əopen Access. @ 2019 R. Kumar et al., published by De Gruyter. (cc) BY License

\section{Introduction}

Pure metals or ceramics have limited application in structural engineering because of the demand of conflicting properties requirements. Some new magnificent advanced composite plates which are made up of functionally graded materials (FGM) are in much demand because of their unique material properties which vary from the surface of one direction to the surface of the other direction. In FGM, an excellent combination of properties which are different from the individual parent materials and lighter in weight used. FGM was first proposed by Bever and Duwez [1] in 1972. Due to their excellent properties, FGMs widely used in the aerospace industry, aeronautical engineering applications, biomedical, electronic chips, heatresisting environment, and many structural applications. Some significant advantages of the FGM are the elimination of delamination mode of failure, residual stresses, high interlinear shear stresses, local stress concentration at the interface or reducing thermal stress, which are the characteristic phenomena observed in laminated composite materials. The research on plates started with the analysis of thin plates using Classical Plate Theory (CPT). Then the first-order shear deformation theory (FSDT) was introduced, whose accuracy is determined by the shear correction factor, which may be difficult to calculate on advanced composites. To avoid such troubles, higher-order shear deformation theories (HSDTs) introduced. However, the majority of these theories avoid transverse extensibility by neglecting the stretching effects. HSDTs further classified as polynomial and non-polynomial. Non-polynomial HSDTs are mainly trigonometric, hyperbolic, algebraic exponential, and logarithmic shape function. Levy M [2], was probably the first to develop a trigonometric theory for the analysis of thick isotropic plates. Touratier [3], used the same function and developed a Sinusoidal Shear Deformation Theory for isotropic laminated plates with five unknowns. Soldatos [4], first used a hyperbolic shape function to develop HSDT for composite plates. Karama et al., [5] developed the first five variable exponential function for the 
plate analysis. Recently, some new transverse shear deformation theories have introduced for additional accuracy. Mantari et al., [6] introduced a new transverse shear deformation theory for composite plates to avoid a shear correction factor under the sinusoidal and uniformly distributed loads. Grover et al., [7] proposed a new inverse hyperbolic shear deformation theory for static buckling analysis of laminated composites the and sandwich plates to provide an accurate solution which is less from computational error. Mantari\& Guedes Soares [8], proposed a new nonpolynomial HSDT for investigation of the static response of composite plates. Aicha Bessaim et al., [9] introduced a new hyperbolic HSDT to improve the accuracy of bending and vibration analysis of a sandwich plate with a thin functionally graded sheet. A new hyperbolic transverse shear deformation theory was introduced by El Meiche et al., [10] to improve the effects of buckling and free vibration analysis of thick FG sandwich plates. Thai and Vo [11], introduced a new sinusoidal transverse shear deformation theory for bending, buckling, and vibration analysis of FG plate to improve the accuracy of the analysis. Mahi et al., [12] developed a new hyperbolic shear transverse theory to avoid the shear correction factor which improved the accuracy of bending and free vibration analysis of isotropic, functionally graded, sandwich and laminated composite. Joshan et al., [13] developed four variables inverse hyperbolic shear deformation theory for analysis of laminated composite plates. Mantari, [15] developed an accurate sinusoidal trigonometric theory for the analysis of the FGM plate and shells. Viola et al., [16] introduced the new shear function for the static analysis of laminated double curved shells by using Generalized Differential Quadrature (GDQ) method. Viola et al., [17], investigated free vibration analysis of double-curved laminated shells and panels and comparison among the FSDT and existing HSDT.

There are many computational methods available for solving the partial differential equation (PDE) and analyzing the composite structures. The generation of a mesh in mesh-based computational methods is a difficult task for any structure, so meshfree methods are an alternative to mesh-based numerical methods. A meshfree method does not require a mesh to discretize the PDE. Some meshless methods depend on background meshes that may support numerical quadrature calculations. Meshfree methods can categorized into two forms for solving the PDE which is known as a weak form and strong form meshfree methods.Regarding weak meshless methods, Nayroles et al., [18] were first to introduce a new diffuse approximation to interpolate through moving least squares into a Galerkin scheme. On the other side, collocation meshfree methods work on the strong form of the PDE. The radial basis func- tion method was first used by Hardy [19] and proposed a simple shape parameter $c=0.815 h$, where ' $h$ ' is the average distance between the closest neighbors. After 20 years, Kansa [20], developed the solution of PDEs. Franke [21] has ranked MQRBF as the best interpolation method based on its accuracy, execution time, and ease of implementation. Cheng [22] investigated subject positive definiteness, error estimate, optimal shape parameter, traditional and effective condition numbers, round-off error, derivatives of interpolator, and the edge effect of multiquadric radial basis functions and its shape parameter. A detailed discussion of RBFs seen in Liu and Gu [23, 24]. While utilizing RBFs, several shape parameters need to determined for better performance Chen et al., [25] investigated free vibration of FGM plate using a local natural neighbor interpolation meshless method based on FSDT. Kumar and Singh, [26] investigated various HSDT for the buckling analysis of FGM plate via the RBF method. Ferreira et al., [27] analysis composite plate by using MQ-RBF based meshfree method. Kumar et al. [28] investigated bending response of FGM plate under patch load by using Wendland RBF. Ferreira et al., [29] used RBF based collocation method for the bending analysis of functionally graded plates. Neves et al., [30] investigated Adaptive Methods for Analysis of Composite Plates with RBF. Roque et al., [31] and Ferreira et al., [32] investigated bending and vibration analysis of composite plates by using RBF based layerwise theory. Singh et al., [33] used the meshfree technique for the analysis of laminated composite and sandwich plates using various RBF. Fantuzzi et al., [34] applied the RBF method for the free vibrations of doubly-curved laminated composite shells and panels by using HSDT.

The typical literature for the buckling and vibration analysis of FGM plates are rare as compared to isotropic, laminated and sandwich plates. Zhu and Liew [35], carried out a free vibration analysis of functionally graded plates using FSDT based on local Kriging meshless technique. Ferreira et al., [36] carried out a free vibration analysis of FGM plate by using the multiquadric radial basis function-based collocation method. Xiang and Xing [37] developed a new FSDT having two independent variables for the free vibrations of a rectangular plate. Zhao et al. [38] implemented an element free kp-Ritz technique to analyze free vibration of functionally graded plates. FSDT is used to analyze transverse shear strain and, mesh-free kernel functions used to approximate the 2-dimensional displacement fields. Vu et al., [39] introduced four variable FSDT for the static and free vibration analysis of FGM plate. Neves et al., [40] investigated static and free vibration analysis of FGM plates by using quasi-3D hyperbolic shear deformation theory with RBF based meshless technique. $\mathrm{Wu}$ 
and Chiu [41], carried RMVT-based meshless collocation and element-free Galerkin technique for the quasi-3D free vibration analysis of FGM plates. Thai and Choi, [42] presented a buckling analysis of FGM plate with four variables by applying closed form solutions. Park and Choi, [43] used two variables FSDT models for bending, buckling, and free vibration analyses of isotropic plates. Buckling analysis of plates under uniaxial and biaxial compression with shear loads was carried out by Bui et al., [44] using the efficient meshfree method. Galerkin meshfree formulation was presented by Sadamoto et al., [45] for buckling analysis of stiffened plates. Zhao et al., [46] carried a buckling analysis of FGM plate based on the element-free kp-Ritz method, and the formulations based on FSDT, also including the effect of the imperfection of initial geometry. Moslemi et al., [47] introduced an analytical solution for the buckling analysis of isotropic plate subjected to uniaxial or biaxial uniformly distributed in-plane load. Isogeometric buckling analysis of FGM was carried out by Yu et al. [48] based on first-order shear deformation theory without considering the effect of shear locking. Chakraverty and Pradhan, [49] used the Rayleigh-Ritz method to investigate the free vibration of $\mathrm{FG}$ plates under various boundary conditions. Talha and Singh [50], carried out bending and vibration analysis of FGM plates by using the FEM method.

FGM plates resting on elastic foundations are one of the most important structural components used in aircraft on runways, footings, mat foundations, and many astronautic and marine structures. Malekzadeh, [51] investigated 3D free vibration of FGM plate resting on foundation, and the material properties vary exponentially through the thickness. Ait Atmane et al., [52] investigated free vibration of FGM plate by using trigonometric HSDT resting on foundation. Sobhy, [53] investigated buckling and free vibration response of FGM sandwich plate resting on foundation. Studies on the buckling and vibrations of porous FGM plates have limited in the literature. Shahsavari et al., [54] investigated free vibration of three types of porosity distribution FGM plate by using quasi-3D hyperbolic theory. Wang et al., [55] investigated non-linear free vibration of FGM plate with porosity effect. Zhao et al., [56] used 3-D exact solution for vibration analysis of three types of porosity distributions FGM plates subject to various boundary conditions. Kiran and Kattimani [57], examined the bending and free vibration of the porous FGM plate by using the finite element method.

In the present work, MQ-RBF based meshfree method implemented for the buckling and free vibration analysis of porous FGM plates with proposed HSDTs on the foundation. The GDE of the FGM plate is developed using the principle of virtual works.MQ-RBF based Meshfree method im-

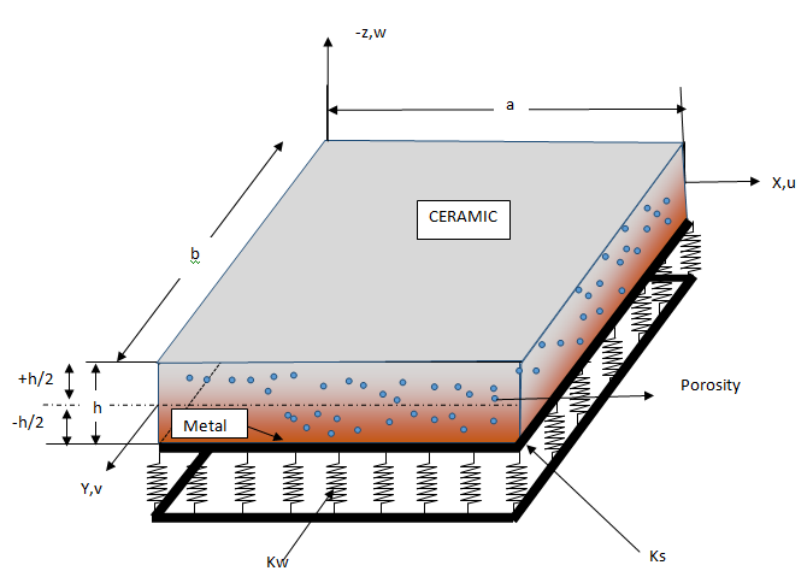

Figure 1: The geometry of a rectangular FGM plate with porosity in the rectangular coordinate system

plemented for discretizing the GDE. The convergence and validation study of a meshfree method for various problems carried out. It noticed that the developed theory results predict closer to the 3-D elasticity results. Some new results for buckling and free vibration analysis of FGM plate in porous media with on foundation are presented using IHHSDT based on five variables ESL theory. The effect of porosity distribution, porosity fraction index, grading index, foundation effect, and span to thickness ratio examined.

\section{Theoretical formulation}

\subsection{Description of the FGP plates}

A functionally graded material plate with dimensions $(a \times$ $\mathrm{b} \times \mathrm{h})$ in the cartesian coordinate system (x-y-z) shown in Figure 1. The mid-plane of the plate considered as the reference plane. The effective materials properties of the FGM plate are uniform across the thickness direction based on the modified power-law in the Voigt model. Three types of porosity distributions along the thickness direction are as follows:

- SCPD: Symmetric center enhanced porosity distribution, [58]

$$
\begin{aligned}
E(z) & =\left(\left[E_{c}-E_{m}\right]\left(\frac{2 z+h}{2 h}\right)^{n}\right. \\
& \left.+E_{m}\right)\left[1-P \cos \left(\frac{\pi z}{h}\right)\right]
\end{aligned}
$$


Table 1: The shear strain functions proposed by different researchers are as under:

\begin{tabular}{cccc}
\hline S.No & Transverse shear strain function $(f(\mathrm{z}))$ & Abbreviation & Proposed \\
\hline 1 & $z \tan ^{-1} h\left(\tanh \left(2 \pi\left(\frac{z}{h}\right)^{2}\right)\right)-\left(\frac{3 \pi z}{2}\right)$ & IHHSDT & New proposed theory \\
2 & $\left(z-\frac{4 z^{3}}{3 h^{2}}\right)$ & HSDT-1 & Reddy [59] \\
3 & $\frac{h}{\pi} \sin \left(\frac{\pi z}{h}\right)$ & HSDT-2 & Touratier, [60] \\
& & & Zenkour, [61] \\
4 & $h \sinh \left(\frac{z}{h}\right)-z \cosh \left(\frac{1}{2}\right)$ & HSDT-3 & Mantari et al., [62] \\
5 & $\sin \left(\frac{\pi z}{h}\right)$ & HSDT-4 & Arya et al., [63] \\
6 & $z \times e^{-2\left(\frac{z}{h}\right)^{2}}$ & HSDT-5 & Karama et al., [64] \\
7 & $z \alpha^{-2(z / h)^{2} / \ln \alpha}$ & HSDT-6 & Aydogdu, [65] \\
8 & $\sin \left(\frac{\pi z}{h}\right) \times e^{\frac{1}{2} \cos \left(\frac{\pi z}{h}\right)}+\frac{\pi z}{2 h}$ & HSDT-7 & Mantari et al., [42] \\
9 & $\sinh { }^{-1}\left(\frac{r z}{h}\right)-z \frac{2 r}{h \sqrt{r^{2}+4}}, r=3$ & HSDT-8 & Grover et al., [66] \\
10 & $h \tan ^{-1}(2 z / h)-z$ & HSDT-9 & Thai et al., [67] \\
11 & $\frac{7}{8} z-\frac{2 z^{3}}{h^{2}}+\frac{2 z^{5}}{h^{4}}$ & HSDT-10 & Nguyen-Xuan et al., [68] \\
12 & $\frac{h}{2} \tanh \left(\frac{2 z}{h}\right)-\frac{4}{3 \cosh h^{2}(1)} \frac{z^{3}}{h^{2}}$ & HSDT-11 & Thai et al., [69] \\
13 & $\tan \left(\frac{m z}{h}\right)+\left\{2 z \cosh ^{2}\left(\frac{1}{2}\right)\right\}$ & HSDT-12 & Singh and Singh, [70] \\
14 & $m=5$ & & Nguyen et al., [71] \\
15 & {$\left[z-\frac{11}{5 h^{2}} z^{3}+\frac{52}{25 h^{4}} z^{5}\right]$} & HSDT-13 & Nguyen et al., [71] \\
\hline
\end{tabular}

- BPD: Bottom enhanced porosity distribution, [58]

$$
\begin{aligned}
E(z) & =\left[\left[E_{c}-E_{m}\right]\left(\frac{2 z+h}{2 h}\right)^{n}+E_{m}\right](1 \\
& \left.-P \cos \left[\frac{\pi}{2}\left(\frac{z}{h}+0.5\right)\right]\right)
\end{aligned}
$$

- TPD: Top enhanced porosity distribution, [58]

$$
\begin{aligned}
E(z) & =\left[\left[E_{c}-E_{m}\right]\left(\frac{2 z+h}{2 h}\right)^{n}+E_{m}\right](1 \\
& \left.-P \cos \left[\frac{\pi}{2}\left(\frac{z}{h}-0.5\right)\right]\right)
\end{aligned}
$$

Where $E$ represents the effective material property (Young's modulus), subscripts $m$ and $c$ represents the metallic and ceramic constituents respectively, $P$ is the porosity fraction $(0<P<1)$ and $\mathrm{P}=0$ means pure FGM plate. ' $n$ ' is exponent governing the material properties along the thickness direction known as grading index. The value of ' $n$ ' equal to 0 and $\infty$ represents a fully ceramic and metal plate, respectively.

\subsection{Displacement field}

The displacement model can represented in terms of generalized transverse $\operatorname{HSDT} f(z)$ and the in-plane displacement $u, v$ and $w$ at any point along the $x$-, y-and $z$-axes can be expressed in terms of the mid-plane displacements $u_{o}, v_{o}, w_{o}$ and rotations $\phi_{x}$ and $\phi_{y}$ which are five unknown displacement functions as :

$$
\begin{aligned}
& u=u_{0}(x, y)-z \frac{\partial w_{0}(x, y)}{\partial x}+f(z) \phi_{x}(x, y) \\
& v=v_{0}(x, y)-z \frac{\partial w_{0}(x, y)}{\partial y}+f(z) \phi_{y}(x, y) \\
& w=w_{0}(x, y)
\end{aligned}
$$

The $f(\mathrm{z})$ represents the transverse shear deformation function which responsible for examining the distribution of the transverse shear strains and stresses along with the thickness. The proposed and existing transverse shear deformation function $f(z)$ listed in Table 1. Researchers are always in search of new theory for better accuracy and stability of results. In the same line, authors of this paper tried to find a new theory which can give a better solution. The function $f(z)$ is chosen in such a way that it satisfies the conditions of continuity and differentiability, such that

$$
\int_{-h / 2}^{h / 2} f(\mathrm{z}) \mathrm{d} z=0 \quad f^{\prime}( \pm h / 2)=0
$$

These conditions are essential for a transverse shear deformation function in order to eliminate the requirement of the shear correction factor. From Figure 2, it can clearly see that the newly proposed theory account for the parabolic variation of transverse shear strains through the span to a thickness of the plate. Furthermore, the proposed theory 


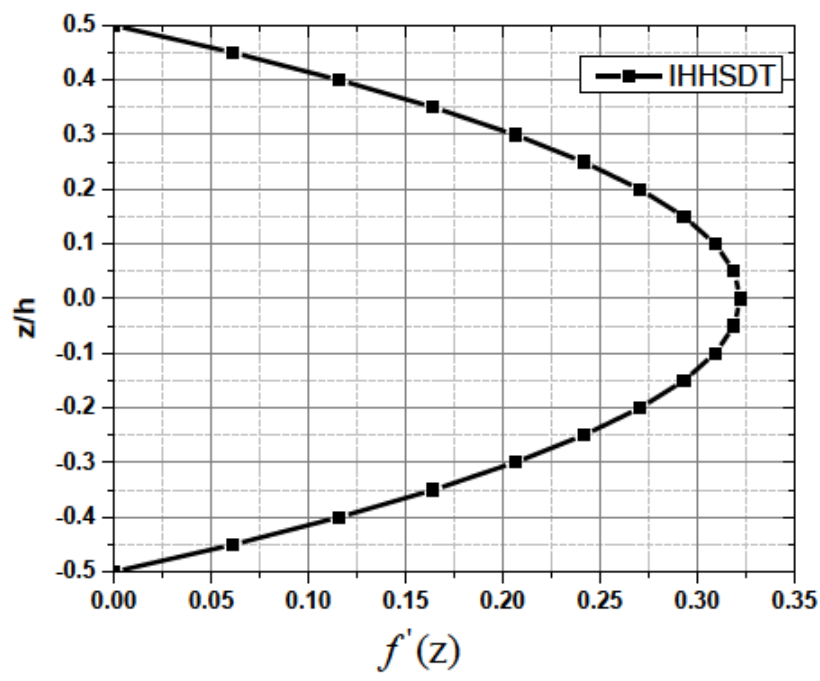

Figure 2: Transverse stress profile of different transverse shear strain function

satisfies the zero traction boundary conditions on the top and bottom surfaces of the plate.

\subsection{Strain-displacement relationships}

According to the linear, small-strain elasticity theory, the strain-displacement relations expressed as:

$$
\begin{gathered}
\varepsilon_{x x}=\frac{\partial u}{\partial x}=\frac{\partial u_{0}}{\partial x}-z \frac{\partial^{2} w_{0}}{\partial x^{2}}+f(z) \frac{\partial \phi_{x}}{\partial x} \\
\varepsilon_{y y}=\frac{\partial v}{\partial y}=\frac{\partial v_{0}}{\partial y}-z \frac{\partial^{2} w_{0}}{\partial y^{2}}+f(z) \frac{\partial \phi_{y}}{\partial y} \\
\gamma_{x y}=\frac{\partial u}{\partial y}+\frac{\partial v}{\partial x}=\frac{\partial u_{0}}{\partial y}+\frac{\partial v_{0}}{\partial x}-2 z \frac{\partial^{2} w_{0}}{\partial x \partial y}+f(z) \frac{\partial \phi_{x}}{\partial y} \\
+f(z) \frac{\partial \phi_{y}}{\partial x} \\
\gamma_{y z}=\frac{\partial v}{\partial z}+\frac{\partial w}{\partial y}=\frac{\partial f(z)}{\partial z} \phi_{y} \\
\gamma_{z x}=\frac{\partial u}{\partial z}+\frac{\partial w}{\partial x}=\frac{\partial f(z)}{\partial z} \phi_{x}
\end{gathered}
$$

\subsection{Constitutive Stress-Strain Relations}

The stress-strain relation using Hook's law concerning the structural axis system (X-Y-Z) for the isotropic FGM plate with $\sigma_{z z}=0$ be expressed as Ferreira et al., [29];

$$
\left\{\begin{array}{l}
\sigma_{x x} \\
\sigma_{y y} \\
\sigma_{x y} \\
\sigma_{y z} \\
\sigma_{z x}
\end{array}\right\}=\left[\begin{array}{ccccc}
\bar{Q}_{11} & \bar{Q}_{12} & 0 & 0 & 0 \\
\bar{Q}_{12} & \bar{Q}_{22} & 0 & 0 & 0 \\
0 & 0 & \bar{Q}_{66} & 0 & 0 \\
0 & 0 & 0 & \bar{Q}_{44} & 0 \\
0 & 0 & 0 & 0 & \bar{Q}_{55}
\end{array}\right]\left\{\begin{array}{c}
\varepsilon_{x x} \\
\varepsilon_{y y} \\
\gamma_{x y} \\
\gamma_{y z} \\
\gamma_{z x}
\end{array}\right\}
$$

Where $\{\sigma\}$ and $\{\varepsilon\}$ are the stress vector and strain vector respectively and $\bar{Q}_{i, j}$ is transformed reduced stiffness matrix.

$$
\begin{aligned}
& \bar{Q}_{11}=\bar{Q}_{22}=\frac{E}{\left(1-v^{2}\right)}, \\
& \bar{Q}_{12}=\frac{v E}{\left(1-v^{2}\right)}, \quad \bar{Q}_{44}=\bar{Q}_{55}=\bar{Q}_{66}=\frac{E}{2(1+v)}
\end{aligned}
$$

\subsection{Governing Differential Equations}

Hamilton's principle is used herein to derive the governing differential equations of the FGM plate along with variationally admissible boundary conditions. The principle can be expressed as

$$
\int_{t_{1}}^{t_{2}} \delta(K E-(U E+V E)) d t=0
$$

Where $\delta$ denotes the variational symbol and $\rho$ is the mass per unit volume.

The strain energy (UE) of the FGM plate is expressed by [72]

$$
\begin{aligned}
U E & =\frac{1}{2} \int_{-\frac{h}{2}}^{\frac{h}{2}} \int_{A}\left(\sigma_{x x} \varepsilon_{x x}\right. \\
& \left.+\sigma_{y y} \varepsilon_{y y}+\sigma_{x y} \gamma_{x y}+\sigma_{y z} \gamma_{y z}+\sigma_{x z} \gamma_{x z}\right) d z d A
\end{aligned}
$$

The kinetic energy (KE) of the FGM plate is expressed by [72]

$$
\begin{aligned}
K E & =\frac{1}{2} \int_{-\frac{h}{2}}^{\frac{h}{2}} \int_{A} \rho\left\{\left(\frac{\partial u}{\partial \tau}\right)^{2}\right. \\
& \left.+\left(\frac{\partial v}{\partial \tau}\right)^{2}+\left(\frac{\partial w}{\partial \tau}\right)^{2}\right\} d z d A
\end{aligned}
$$

The potential energy (PE) due to the in-plane mechanical loading of the FGM plate is expressed by

$$
V E=\frac{1}{2} \int_{A}\left\{N_{x}^{b}\left(\frac{\partial w}{\partial x}\right)^{2}\right.
$$




$$
\left.+N_{y}^{b}\left(\frac{\partial w}{\partial y}\right)^{2}+2 N_{x y}^{b}\left(\frac{\partial w}{\partial x}\right)\left(\frac{\partial w}{\partial y}\right)\right\} d A
$$

The potential energy $\left(\mathrm{VE}_{f}\right)$ of the elastic foundation is expressed by [72]

$$
\begin{aligned}
V E_{f} & =\frac{1}{2}\left(\int_{A} K_{w} w^{2}\right. \\
& \left.-K_{S}\left(\left(\frac{\partial w}{\partial x}\right)^{2}+\left(\frac{\partial w}{\partial y}\right)^{2}\right) d A\right)
\end{aligned}
$$

Considering displacement-strain relations and stressstrain relations, and applying integration by parts and the fundamental lemma of variational calculus and collecting the coefficients of $\delta u_{0}, \delta v_{0}, \delta w_{0}, \delta \phi_{x}$, and $\delta \phi_{y}$. The governing differential equations of the plate obtained as:

$$
\begin{aligned}
& \delta u_{0}: \quad \frac{\partial N_{x x}}{\partial x}+\frac{\partial N_{x y}}{\partial y}=I_{0} \frac{\partial^{2} u_{0}}{\partial \tau^{2}}-I_{1} \frac{\partial^{3} w_{0}}{\partial x \partial \tau^{2}} \\
& +I_{3} \frac{\partial^{2} \phi_{x}}{\partial \tau^{2}} \\
& \delta v_{0}: \quad \frac{\partial N_{x y}}{\partial x}+\frac{\partial N_{y y}}{\partial y}=I_{0} \frac{\partial^{2} v_{0}}{\partial \tau^{2}}-I_{1} \frac{\partial^{3} w_{0}}{\partial y \partial \tau^{2}} \\
& +I_{3} \frac{\partial^{2} \phi_{y}}{\partial \tau^{2}} \\
& \delta w_{0}: \frac{\partial^{2} M_{x x}}{\partial x^{2}}+\frac{\partial^{2} M_{y y}}{\partial y^{2}}+2 \frac{\partial^{2} M_{x y}}{\partial x \partial y}+K_{w} w_{0} \\
& -K_{s}\left(\frac{\partial^{2} w_{0}}{\partial x^{2}}+\frac{\partial^{2} w_{0}}{\partial y^{2}}\right)=N_{x x}^{b} \frac{\partial^{2} w}{\partial x^{2}}+N_{y y}^{b} \frac{\partial^{2} w}{\partial y^{2}} \\
& +2 N_{x y}^{b} \frac{\partial^{2} w}{\partial x \partial y}+I_{0} \frac{\partial^{2} w_{0}}{\partial t^{2}}+I_{1}\left(\frac{\partial^{3} u_{0}}{\partial x \partial \tau^{2}}+\frac{\partial^{3} v_{0}}{\partial y \partial \tau^{2}}\right) \\
& -I_{2}\left(\frac{\partial^{4} w_{0}}{\partial x^{2} \partial \tau^{2}}+\frac{\partial^{4} w_{0}}{\partial y^{2} \partial \tau^{2}}\right) \\
& +I_{4}\left(\frac{\partial^{3} \phi_{x}}{\partial x \partial \tau^{2}}+\frac{\partial^{3} \phi_{y}}{\partial y \partial \tau^{2}}\right) \\
& \delta \phi_{x}: \quad \frac{\partial M_{x x}^{f}}{\partial x}+\frac{\partial M_{x y}^{f}}{\partial y}-Q_{x}^{f}=I_{3} \frac{\partial^{2} u_{0}}{\partial \tau^{2}}-I_{4} \frac{\partial^{3} w_{0}}{\partial x \partial \tau^{2}} \\
& +I_{5} \frac{\partial^{2} \phi_{x}}{\partial \tau^{2}} \\
& \delta \phi_{y}: \quad \frac{\partial M_{x y}^{f}}{\partial x}+\frac{\partial M_{y y}^{f}}{\partial y}-Q_{y}^{f}=I_{3} \frac{\partial^{2} v_{0}}{\partial \tau^{2}}-I_{4} \frac{\partial^{3} w_{0}}{\partial y \partial \tau^{2}} \\
& +I_{5} \frac{\partial^{2} \phi_{y}}{\partial \tau^{2}}
\end{aligned}
$$

Where, $N_{x x}^{b}$ and $N_{y y}^{b}$ are the in-plane axial forces and $N_{x y}^{b}=$ $N_{y x}^{b}$ is the in-plane shear force is listed in Figure 3. In equ. 18 if $N_{x x}^{b}=1, N_{y y}^{b}=0, N_{x y}^{b}=0$ is uniaxial loading, $N_{x x}^{b}=1, N_{y y}^{b}=1, N_{x y}^{b}=0$ is biaxial loading, and $N_{x x}^{b}=0, N_{y y}^{b}=0, N_{x y}^{b}=1$ is pure shear loading. $K s$ and $K w$ are the equivalent shear and Winkler foundation parameters. The axial force resultants, $N_{i j}$ the bending moment resultants $M_{i j}$, the additional moment resultants related to the transverse shear function $M_{i j}^{f}$ and the transverse shear force resultants $Q_{x}^{f}$ and $Q_{y}^{f}$ expressed as:

$$
\begin{gathered}
N_{i j}, M_{i j}, M_{i j}^{f}=\int_{-h / 2}^{+h / 2}\left(\sigma_{i j}, z \sigma_{i j}, f(z) \sigma_{i j}\right) d z \\
Q_{x}^{f}, Q_{y}^{f}=\int_{-h / 2}^{+h / 2}\left(\sigma_{x z}, \sigma_{y z}\right)\left(\frac{\partial f(z)}{\partial z}\right) d z
\end{gathered}
$$

\subsection{Governing differential equation in terms of displacement}

The governing differential Eqs (16) - (20) are present in terms of displacement components using Eqs 6 and 10 and finally expressed as:

$$
\begin{aligned}
& \left(A_{11} \frac{\partial^{2} u_{0}}{\partial x^{2}}+A_{66} \frac{\partial^{2} u_{0}}{\partial y^{2}}\right) \\
& +\left(A_{12} \frac{\partial^{2} v_{0}}{\partial x \partial y}+A_{66} \frac{\partial^{2} v_{0}}{\partial x \partial y}\right) \\
& +\left(-B_{11} \frac{\partial^{3} w_{0}}{\partial x^{3}}-B_{12} \frac{\partial^{3} w_{0}}{\partial x \partial y^{2}}-2 B_{66} \frac{\partial^{3} w_{0}}{\partial x \partial y^{2}}\right) \\
& +\left(E_{11} \frac{\partial^{2} \phi_{x}}{\partial x^{2}}+E_{66} \frac{\partial^{2} \phi_{x}}{\partial y^{2}}\right)+\left(E_{12} \frac{\partial^{2} \phi_{y}}{\partial x \partial y}+E_{66} \frac{\partial^{2} \phi_{y}}{\partial x \partial y}\right) \\
& =I_{0} \frac{\partial^{2} u_{0}}{\partial \tau^{2}}-I_{1} \frac{\partial^{3} w_{0}}{\partial x \partial \tau^{2}}+I_{3} \frac{\partial^{2} \phi_{x}}{\partial \tau^{2}} \\
& \left(A_{66} \frac{\partial^{2} u_{0}}{\partial x \partial y}+A_{12} \frac{\partial^{2} u_{0}}{\partial x \partial y}\right)+\left(A_{66} \frac{\partial^{2} v_{0}}{\partial x^{2}}+A_{22} \frac{\partial^{2} v_{0}}{\partial y^{2}}\right) \\
& +\left(-2 B_{66} \frac{\partial^{3} w_{0}}{\partial x^{2} \partial y}-B_{12} \frac{\partial^{3} w_{0}}{\partial x^{2} \partial y}-B_{22} \frac{\partial^{3} w_{0}}{\partial y^{3}}\right) \\
& +\left(\left(E_{12}+E_{66}\right) \frac{\partial^{2} \phi_{x}}{\partial x \partial y}\right)+\left(E_{66} \frac{\partial^{2} \phi_{y}}{\partial x^{2}}+E_{22} \frac{\partial^{2} \phi_{y}}{\partial y^{2}}\right) \\
& =I_{0} \frac{\partial^{2} v_{0}}{\partial \tau^{2}}-I_{1} \frac{\partial^{3} w_{0}}{\partial y \partial \tau^{2}}+I_{3} \frac{\partial^{2} \phi_{y}}{\partial \tau^{2}} \\
& +\left(B_{11} \frac{\partial^{3} u_{0}}{\partial x^{3}}+\left(B_{12}+2 B_{66}\right) \frac{\partial^{3} u_{0}}{\partial x \partial y^{2}}\right) \\
& +\left(B_{22} \frac{\partial^{3} v_{0}}{\partial y^{3}}+\left(B_{12}+2 B_{66}\right) \frac{\partial^{3} v_{0}}{\partial x^{2} \partial y}\right) \\
& -\left(D_{11} \frac{\partial^{4} w_{0}}{\partial x^{4}}+\left(2 D_{12}+4 D_{66}\right) \frac{\partial^{4} w_{0}}{\partial x^{2} \partial y^{2}}+D_{22} \frac{\partial^{4} w_{0}}{\partial y^{4}}\right)
\end{aligned}
$$




$$
\begin{aligned}
& +\left(F_{11} \frac{\partial^{3} \phi_{x}}{\partial x^{3}}+\left(F_{12}+2 F_{66}\right) \frac{\partial^{3} \phi_{x}}{\partial x \partial y^{2}}\right) \\
& +\left(F_{22} \frac{\partial^{3} \phi_{y}}{\partial y^{3}}+\left(F_{12}+2 F_{66}\right) \frac{\partial^{3} \phi_{y}}{\partial x^{2} \partial y}\right)+K_{w} w_{0} \\
& -K_{s}\left(\frac{\partial^{2} w_{0}}{\partial x^{2}}+\frac{\partial^{2} w_{0}}{\partial y^{2}}\right)=N_{x x}^{b} \frac{\partial^{2} w}{\partial x^{2}}+N_{y y}^{b} \frac{\partial^{2} w}{\partial y^{2}} \\
& +2 N_{x y}^{b} \frac{\partial^{2} w}{\partial x \partial y}+I_{0} \frac{\partial^{2} w_{0}}{\partial t^{2}}+I_{1}\left(\frac{\partial^{3} u_{0}}{\partial x \partial \tau^{2}}+\frac{\partial^{3} v_{0}}{\partial y \partial \tau^{2}}\right) \\
& -I_{2}\left(\frac{\partial^{4} w_{0}}{\partial x^{2} \partial \tau^{2}}+\frac{\partial^{4} w_{0}}{\partial y^{2} \partial \tau^{2}}\right)+I_{4}\left(\frac{\partial^{3} \phi_{x}}{\partial x \partial \tau^{2}}+\frac{\partial^{3} \phi_{y}}{\partial y \partial \tau^{2}}\right) \\
& \left(E_{11} \frac{\partial^{2} u_{0}}{\partial x^{2}}+E_{66} \frac{\partial^{2} u_{0}}{\partial y^{2}}\right)+\left(E_{12} \frac{\partial^{2} v_{0}}{\partial x \partial y}+E_{66} \frac{\partial^{2} v_{0}}{\partial x \partial y}\right) \\
& +\left(-F_{11} \frac{\partial^{3} w_{0}}{\partial x^{3}}-\left(F_{12}+2 F_{66}\right) \frac{\partial^{3} w_{0}}{\partial x \partial y^{2}}\right) \\
& +\left(H_{11} \frac{\partial^{2} \phi_{x}}{\partial x^{2}}+H_{66} \frac{\partial^{2} \phi_{x}}{\partial y^{2}}-A_{55} \phi_{x}\right) \\
& +\left(\left(H_{12}+H_{66}\right) \frac{\partial^{2} \phi_{y}}{\partial x \partial y}+H_{22} \frac{\partial^{2} \phi_{y}}{\partial y^{2}}\right)=I_{3} \frac{\partial^{2} u_{0}}{\partial \tau^{2}} \\
& -I_{4} \frac{\partial^{3} w_{0}}{\partial x \partial \tau^{2}}+I_{5} \frac{\partial^{2} \phi_{x}}{\partial \tau^{2}} \\
& \left(E_{66} \frac{\partial^{2} u_{0}}{\partial x \partial y}+E_{12} \frac{\partial^{2} u_{0}}{\partial x \partial y}\right) \\
& +\left(+E_{66} \frac{\partial^{2} v_{0}}{\partial x^{2}}+E_{22} \frac{\partial^{2} v_{0}}{\partial y^{2}}\right) \\
& +\left(-\left(F_{12}+F_{66}\right) \frac{\partial^{3} w_{0}}{\partial y \partial x^{2}}-F_{22} \frac{\partial^{3} w_{0}}{\partial y^{3}}\right) \\
& +\left(H_{66} \frac{\partial^{2} \phi_{x}}{\partial x \partial y}+H_{12} \frac{\partial^{2} \phi_{x}}{\partial x \partial y}\right) \\
& +\left(H_{66} \frac{\partial^{2} \phi_{y}}{\partial x^{2}}+H_{22} \frac{\partial^{2} \phi_{y}}{\partial y^{2}}-A_{44} \phi_{y}\right)=I_{3} \frac{\partial^{2} v_{0}}{\partial \tau^{2}} \\
& -I_{4} \frac{\partial^{3} w_{0}}{\partial y \partial \tau^{2}}+I_{5} \frac{\partial^{2} \phi_{y}}{\partial \tau^{2}}
\end{aligned}
$$

The stiffness coefficients for the FGM plate are taken as:

$$
\begin{aligned}
& A_{i j}, B_{i j}, D_{i j}, E_{i j}, F_{i j}, H_{i j} \\
& =\int_{-h / 2}^{h / 2}\left\{\left(\frac{E(z)}{1-v^{2}}\right)\left(1, z, z^{2}, f(z), z f(z), f^{2}(z)\right)\right\} d z
\end{aligned}
$$$$
i, j=1,2,6
$$

$$
A_{i j}=\int_{-h / 2}^{h / 2}\left\{\left(\frac{E(z)}{2(1+v)}\right)\left(\frac{\partial f(z)}{\partial z}\right)^{2}\right\} d z \quad i, j=4,5
$$

$I_{0}, I_{1}, I_{2}, I_{3}, I_{4}, I_{5}$ are mass inertias which is expressed as

$$
I_{0}, I_{1}, I_{2}, I_{3}, I_{4}, I_{5}
$$

$$
=\int_{h_{1}}^{h_{2}} \rho(z)\left(1, z, z^{2}, f(z), z f(z), f^{2}(z)\right) d z
$$

\subsection{Simply supported boundary condition SS boundary condition}

$$
\begin{aligned}
& x=0, \\
& a: N_{x x}=0, v_{0}=0, w_{0}=0, M_{x x}=0, \phi_{y}=0 \\
& y=0, \\
& b: u_{0}=0, N_{y y}=0, w_{0}=0, \phi_{x}=0, M_{y y}=0
\end{aligned}
$$

\section{Solution methodology}

The governing equations, along with the appropriate boundary conditions are solved using multi quadratic radial basis functions RBF based meshfree method. For the present analysis, MQ- RBF is taken and which expressed as:

$$
\begin{aligned}
& \text { Multiquadrics function (MQ-RBF) } g=\left(r^{2}+c^{2}\right)^{m} ; \\
& c=\alpha \cdot \sqrt{\left(\frac{a}{n_{x}}\right)^{2}}+\left(\frac{b}{n_{y}}\right)^{2}
\end{aligned}
$$

Where, $r=\left\|X-X_{j}\right\|=\sqrt{\left(x-x_{j}\right)^{2}+\left(y-y_{j}\right)^{2}}$, ' $m$ ' and ' $c$ ' are shape parameters. $n_{x}$ and $n_{y}$ are a number of divisions (number of nodes-1) along with the ' $a$ ' and ' $b$ ' respectively. $\alpha$ is a constant that governs the value of ' $c$ ' for interior and boundary nodes for MQ-RBF. Optimization of the shape parameter has been discussed by Singh and Shukla [87]. For the present investigation, a MATLAB code is developed to acquire the solution. First, the convergence of results was checked by shifting the shape parameter. When convergence is confirmed and stable, the accuracy was likewise checked. Also, given stability and accuracy, the authors proposed the given shape parameter. A 2-D rectangular with any arbitrary boundary having NB boundary nodes and NI domain interior nodes subjected to compressive in-plane forces and shear distributed forces is shown in Figure 3.

\subsection{Discretization of Governing Differential Equations}

The unknown field variables $u_{0}, v_{0}, w_{0}, \phi_{x}$ and $\phi_{y}$ appearing in governing differential equations can interpolated in 


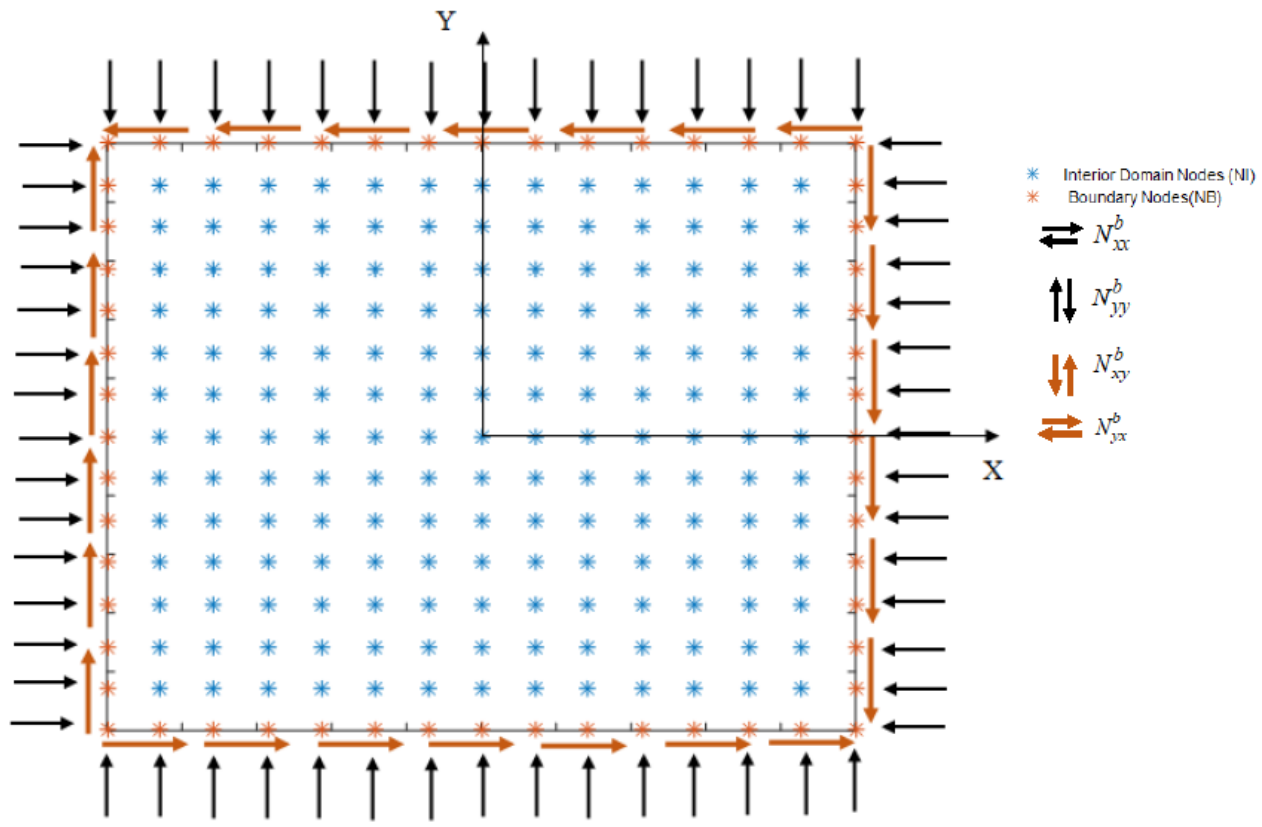

Figure 3: An arbitrary 2-D domain with boundary and domain interior nodes in the form of scattered data subjected to compressive in-plane forces and shear distributed forces

the form of the radial distance between nodes using the following expression:

$$
\begin{aligned}
& u_{0}(x, y)=\sum_{j=1}^{N} \alpha_{j}^{u_{0}} g\left(\left\|X-X_{j}\right\|, c\right) \\
& v_{0}(x, y)=\sum_{j=1}^{N} \alpha_{j}^{v_{0}} g\left(\left\|X-X_{j}\right\|, c\right) \\
& w_{0}(x, y)=\sum_{j=1}^{N} \alpha_{j}^{w_{0}} g\left(\left\|X-X_{j}\right\|, c\right) \\
& \phi_{x}(x, y)=\sum_{j=1}^{N} \alpha_{j}^{\varphi_{x}} g\left(\left\|X-X_{j}\right\|, c\right) \\
& \phi_{y}(x, y)=\sum_{j=1}^{N} \alpha_{j}^{\varphi_{y}} g\left(\left\|X-X_{j}\right\|, c\right)
\end{aligned}
$$

Where, $N$ is a total number of nodes, which is equal to the summation of boundary nodes NB and domain interior nodes NI, $\alpha_{j}^{u}$ is unknown coefficient, $g\left(\left\|X-X_{j}\right\|, c\right)$ is radial basis function, $\left\|X-X_{j}\right\|$ is the distance between the nodes.

The governing differential equations are discretized and finally expressed in compact matrix form as:

$$
\left[[\mathrm{K}]_{\mathrm{L}}+[\mathrm{K}]_{\mathrm{fL}}+\lambda[\mathrm{K}]_{\mathrm{G}}\right]\{\delta\}=\chi \omega^{2}[\mathrm{M}]\{\delta\}
$$

Here,

For free vibration analysis: $\quad \lambda=0, \chi=1$

For buckling analysis: $\lambda=1, \chi=0$

Where,

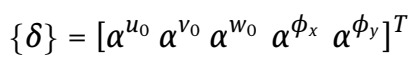

$$
[K]_{L}=\left[\begin{array}{lll}
{\left[K_{1 u}^{l}\right]_{(N I, N)}} & {\left[K^{l}{ }_{1 v}\right]_{(N I, N)}} & \cdots \\
{\left[K^{l}{ }_{2 u}\right]_{(N I, N)}} & {\left[K^{l}{ }_{2 v}\right]_{(N I, N)}} & \cdots \\
{\left[K^{l}{ }_{3 u}\right]_{(N I, N)}} & {\left[K^{l}{ }_{3 v}\right]_{(N I, N)}} & \cdots \\
{\left[K^{l}{ }_{4 u}\right]_{(N I, N)}} & {\left[K^{l}{ }_{4 v}\right]_{(N I, N)}} & \cdots \\
{\left[K^{l}{ }_{5 u}\right]_{(N I, N)}} & {\left[K^{l}{ }_{5 v}\right]_{(N I, N)}} & \cdots
\end{array}\right.
$$

$\left[K_{1 w}^{l}\right]_{(N I, N)} \quad\left[K_{1 \phi_{x}}^{l}\right]_{(N I, N)} \quad\left[K_{1 \phi_{y}}^{l}\right]_{(N I, N)}$

$\left[K^{l}{ }_{2 w}\right]_{(N I, N)} \quad\left[K_{2 \phi_{x}}^{l}\right]_{(N I, N)} \quad\left[K_{2 \phi_{y}}^{l}\right]_{(N I, N)}$

$\left[K^{l}{ }_{3 w}\right]_{(N I, N)} \quad\left[K_{3 \phi_{x}}^{l}\right]_{(N I, N)} \quad\left[K^{l}{ }_{3 \phi_{y}}\right]_{(N I, N)}$

$\left[K^{l}{ }_{4 w}\right]_{(N I, N)} \quad\left[K^{l}{ }_{4 \phi_{x}}\right]_{(N I, N)} \quad\left[K_{4 \phi_{y}}^{l}\right]_{(N I, N)}$

$\left.\left[K^{l}{ }_{5 w}\right]_{(N I, N)} \quad\left[K_{5 \phi_{x}}^{l}\right]_{(N I, N)} \quad\left[K_{5 \phi_{y}}^{l}\right]_{(N I, N)}\right]_{(5 \times N I, 5 \times N)}$

$$
[K]_{G}=\left[\begin{array}{ccccc}
{[0]} & {[0]} & {[0]} & {[0]} & {[0]} \\
{[0]} & {[0]} & {[0]} & {[0]} & {[0]} \\
{[0]} & {[0]} & {\left[G_{3 w_{0}}^{l}\right]} & {[0]} & {[0]} \\
{[0]} & {[0]} & {[0]} & {[0]} & {[0]} \\
{[0]} & {[0]} & {[0]} & {[0]} & {[0]}
\end{array}\right]_{(5 \times N I, 5 \times N)}
$$

$[M]=-\omega^{2} \sum_{i=1}^{N I} \sum_{j=1}^{N}\left[\begin{array}{ccc}{\left[I_{0} g_{(i, j)}\right]} & {[0]} & \cdots \\ {[0]} & {\left[I_{0} g_{(i, j)}\right]} & \cdots \\ {\left[I_{1} \frac{\partial g_{(i, j)}}{\partial x}\right]} & {\left[I_{0} \frac{\partial g_{(i, j)}}{\partial y}\right]} & \ldots \\ {\left[I_{3} g_{(i, j)}\right]} & {[0]} & \ldots \\ {[0]} & {\left[I_{3} g_{(i, j)}\right]} & \cdots\end{array}\right.$ 
Table 2: Mechanical properties of metallic and ceramic materials considered

\begin{tabular}{ccccc}
\hline \multirow{2}{*}{ Types of Functionally graded material } & \multicolumn{3}{c}{ Properties } \\
\cline { 3 - 5 } & & $\mathrm{E}(\mathrm{GPa})$ & $\rho\left(\mathrm{kg} / \mathrm{m}^{3}\right)$ & $v$ \\
\hline FGM-1 & Metal (Al) & 70 & 2702 & 0.3 \\
& Ceramic (Al203) & 380 & 3800 & 0.3 \\
FGM-2 & Metal(Al) & 70 & 2702 & 0.3 \\
& Ceramic (ZrO2) & 151 & 3000 & 0.3 \\
FGM-3 & Metal(Ti-6Al-4V) & 105.7 & 4429 & 0.298 \\
& Ceramic (aluminum oxide) & 320.2 & 3750 & 0.26 \\
FGM-4 & Stainless steel & 201.04 & 8166 & 0.3262 \\
& Silicon nitride & 348.43 & 2370 & 0.2400 \\
\hline
\end{tabular}

Table 3: Convergence and validation study of the critical buckling parameter $\bar{N}=N_{x} d^{2} / \pi^{2}\left(E h^{3} / 12\left(1-v^{2}\right)\right)$ of an isotropic square plate under uniaxial loading $(\mathrm{a}=\mathrm{b}=1)$

\begin{tabular}{ccccccc}
\hline Theory & \multicolumn{3}{c}{ 'a/h' } & Diff\% & 5 & $\begin{array}{c}\text { Avg. } \\
\text { Diff\% }\end{array}$ \\
\cline { 2 - 4 } & 20 & Diff\% & 10 & & & - \\
Rao and Srinivas, [73] & 3.911 & 0 & 3.741 & - & 3.15 & - \\
Uymaz and Aydogdu [74] & 3.9499 & 0.99 & 3.8099 & 1.84 & 3.3299 & 5.71 \\
Present result $(11 \times 11$ nodes) & 3.973 & 1.59 & 3.824 & 2.22 & 3.317 & 5.30 \\
Present result $(13 \times 13$ nodes) & 3.961 & 1.28 & 3.811 & 1.87 & 3.303 & 4.86 \\
Present result $(15 \times 15$ nodes) & 3.955 & 1.13 & 3.804 & 1.68 & 3.296 & 4.63 \\
Present result $(17 \times 17$ nodes) & 3.952 & 1.05 & 3.8 & 1.58 & 3.292 & 4.51 \\
\hline
\end{tabular}

Where Avg. Diff $\%=$ Abs ((Present result- 3D result)/ 3D result) $\times 100$

$$
\left.\begin{array}{ccc}
{\left[-I_{0} \frac{\partial g_{(i, j)}}{\partial x}\right]} & {\left[I_{3} g_{(i, j)}\right]} & {[0]} \\
{\left[-I_{0} \frac{\partial g_{(i, j)}}{\partial y}\right]} & {[0]} & {\left[I_{3} g_{(i, j)}\right]} \\
{\left[I_{0} g_{(i, j)}-I_{2} \frac{\partial^{2} g_{(i, j)}}{\partial x^{2}}-I_{2} \frac{\partial^{2} g_{(i, j)}}{\partial y^{2}}\right]} & {\left[I_{4} \frac{\partial^{2} g_{(i, j)}}{\partial x^{2}}\right]} & {\left[I_{4} \frac{\partial^{2} g_{(i, j)}}{\partial y^{2}}\right]} \\
{\left[-I_{4} \frac{\partial g_{(i, j)}}{\partial x}\right]} & {\left[I_{5} g_{(i, j)}\right]} & {[0]} \\
{\left[-I_{4} \frac{\partial g_{(i, j)}}{\partial y}\right]} & {[0]} & {\left[I_{5} g_{(i, j)}\right]}
\end{array}\right]_{(5 \times N I, 5 \times N)}
$$

\section{Numerical results and discussions}

To validate the accuracy, stability, and efficiency of the proposed theory with MQ-RBF based meshfree method, a significant number of numerical examples presented and discussed to verify the effectiveness of the proposed IHHSDT considered firstly. Based on the convergence study, a 15×15 node used throughout the study. Then, the influence of some critical parameters, including boundary condition, material property distribution, elastic foundation, porosity volume fraction behaviors of the FGM plate discussed in detail. The equations solved by developing a computer program using MATLAB (2015) to obtain the results. Four types of FG Plates used in this study and their material properties listed in Table 2. The equivalent shear $(K s)$ and Winkler $(K w)$ parameters considered throughout the study and which defined as $K s=\frac{k_{s} 12\left(1-v^{2}\right)}{E_{m} h^{3}} \& K w=\frac{k w 12\left(1-v^{2}\right)}{E_{m} h^{3}}$

\subsection{Convergence and Validation study}

In order to check the accuracy, stability and validation purpose of the MQ-RBF method and proposed IHHSDT for the analyses of the buckling and free vibration of the FGM plate, the present results compared with several results available in the open literature. The equations solved by developing a computer program using MATLAB (2015) to obtain the results.

For the accuracy and validation purpose of the present method and proposed theory, the first problems carried out for a simply supported isotropic plate. The numerical results for the uniaxial critical buckling parameter with various spans to thickness ratios were examined in Table 3. It can be observed that the present results are a good agreement with the analytical 3-D solution given by Rao and Srinivas, [73] with the deviation is up to $5 \%$.and also good agreement with the 3D buckling solutions using the Ritz method by Uymaz and Aydogdu [74]. The present results 
Table 4: Convergence and comparison study of non-dimensional frequency parameter $\bar{\omega}=\omega a^{2} / h \sqrt{\rho_{c} / E_{c}}$ of five modes for FGM-1 square plate $(a / b=1, a / h=10, ' n '=1)$

\begin{tabular}{|c|c|c|c|c|c|c|c|}
\hline \multirow[t]{2}{*}{ Modes } & \multicolumn{6}{|c|}{ Number of nodes } & \multirow[t]{2}{*}{ Jin et al. [75] } \\
\hline & $7 \times 7$ & $9 \times 9$ & $11 \times 11$ & $13 \times 13$ & $15 \times 15$ & $17 \times 17$ & \\
\hline Mode 1 & 4.511 & 4.465 & 4.446 & 4.436 & 4.430 & 4.427 & 4.428 \\
\hline Mode 2 & 10.865 & 10.720 & 10.648 & 10.610 & 10.588 & 10.575 & 10.630 \\
\hline Mode 3 & 10.865 & 10.720 & 10.648 & 10.610 & 10.588 & 10.575 & 10.630 \\
\hline Mode 4 & 15.623 & 15.694 & 15.763 & 15.821 & 15.868 & 15.907 & 16.200 \\
\hline Mode 5 & 15.623 & 15.694 & 15.763 & 15.821 & 15.868 & 15.907 & 16.200 \\
\hline
\end{tabular}

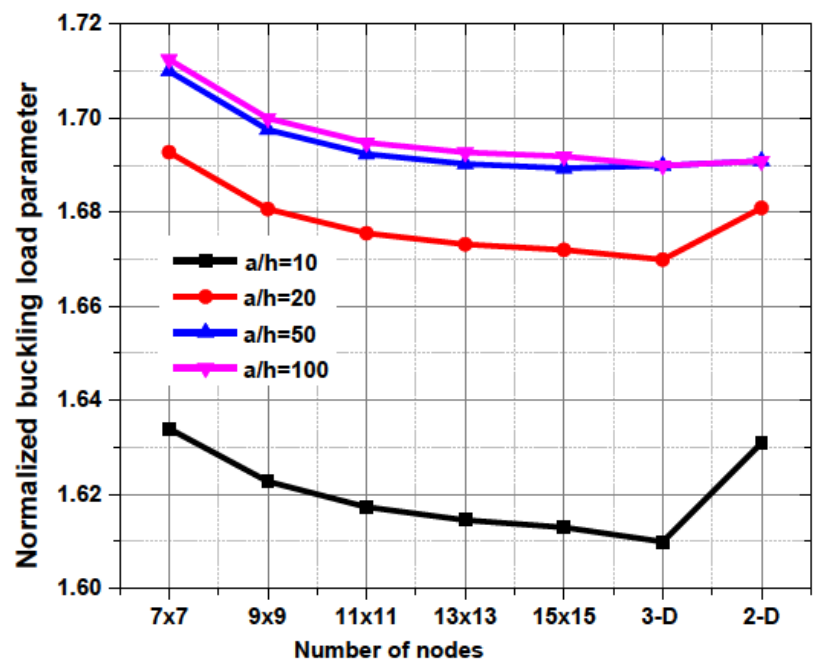

Figure 4: Convergence study of with effect of span to thickness ratio of an FGM-4 plate under biaxial loading $(a=b=1, n=0.5)$

are closer to the Rao and Srinivas, [73] as compared to the Rao and Srinivas, [73].

Figure 4 represents the convergence and validation study of critical buckling parameter of SS FGM plate from thick to thin plate with ' $n$ ' $=1$ considered. It can be observed that convergence of present results with effect of power index is achieved after $13 \times 13$ nodes and validated with the 3-D and elasticity solution given by B. Uymaz, M. Aydogdu [74] with the deviation is up to $2 \%$. It observed that present converged results are closer to 3-D results, which is better than 2-D results.

The convergence and validation study of the nondimensional frequency parameter of five modes for SS FGM-1 square plate presented in Table 4. It can be seen that the present results obtained by proposed theory and method is convergence within $2 \%$ at $15 \times 15$ nodes and shows good agreement with the 3-D solutions by Jin et al., [75].

Figure 5 shows the normalized fundamental frequency parameter from thick to thin FGM-2 plate. The non-dimensional frequency parameter $\varpi=$

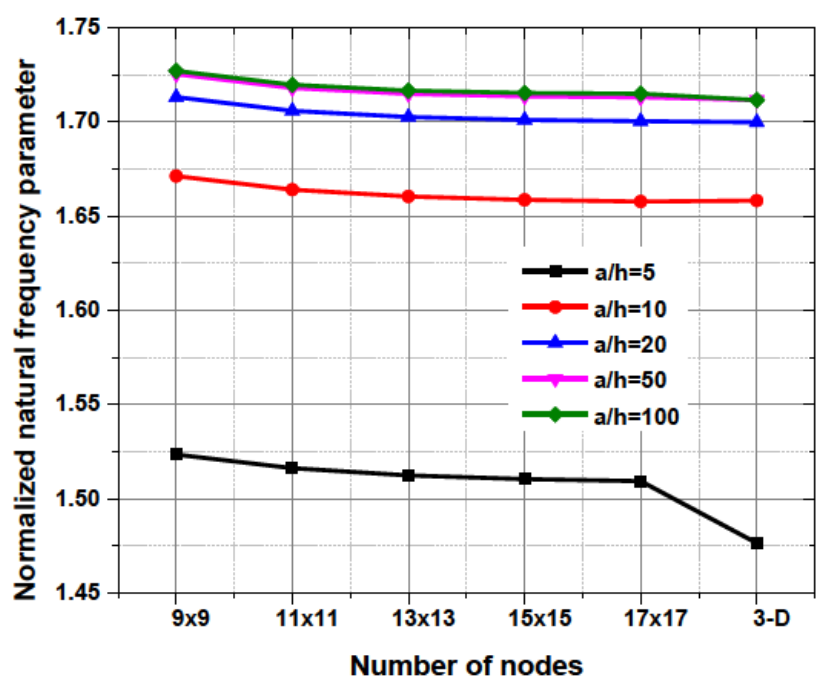

Figure 5: Convergence and validation study of non-dimensional frequency parameter of SS FGM-2 plate with different span to thickness ratio $(a=b=1, n=1)$

$\omega h \sqrt{12\left(1-v^{2}\right) \rho_{c} a^{2} b^{2} /(p i)^{4} h^{2} E_{c}}$ is taken. It can be observed that present results are achieved good convergence after $15 \times 15$ nodes and is predict near to the 3 -D exact solution of Uymaz and Aydogdu [76] from thick to the thin plate. So, it can conclude that the present method and proposed theory is sufficiently accurate for its acceptability for buckling and free vibration analysis of FGM plate and the nodes distribution is $15 \times 15$ nodes can give the results with excellent accuracy and is intensity in its computing time and acceptable for the further analysis.

\subsection{Comparison study}

In this section, the present method results compared with several method results available in the open literature and proposed theory is compared with the 3D solution [76] and the other five variables HSDT theories carried out with the same method to determine their accuracy and applicabil- 
Table 5: Convergence study of $\bar{N}=N_{x} a^{2} / \pi^{2}\left(E h^{3} / 12\left(1-v^{2}\right)\right)$ with effect of grading index of SS FGM-4 under uniaxial loading (a=b=1, a/h=10)

\begin{tabular}{ccccccc}
\hline Theory & \multicolumn{5}{c}{ Grading index ' $n$ ' } & \multirow{2}{*}{ Avg. Diff \% } \\
\cline { 2 - 5 } & 0 & 0.5 & 1 & 5 & 10 & \\
\hline 3D solution [77] & 3.8099 & 3.2299 & 2.9899 & 2.6699 & 2.5699 & 0.34 \\
IHHSDT & 3.8161 & 3.2292 & 3.0083 & 2.6883 & 2.5751 & 0.35 \\
HSDT-1 & 3.8164 & 3.2293 & 3.0087 & 2.6886 & 2.5756 & 0.35 \\
HSDT-2 & 3.8167 & 3.2294 & 3.0088 & 2.6883 & 2.5755 & 1.09 \\
HSDT-3 & 3.8402 & 3.2483 & 3.0278 & 2.7144 & 2.5991 & 0.35 \\
HSDT-4 & 3.8167 & 3.2296 & 3.0088 & 2.6883 & 2.5755 & 0.47 \\
HSDT-5 & 3.8208 & 3.2328 & 3.0121 & 2.6932 & 2.5796 & 0.38 \\
HSDT-6 & 3.818 & 3.2308 & 3.0099 & 2.6892 & 2.5766 & 1.10 \\
HSDT-7 & 3.8406 & 3.2485 & 3.0282 & 2.714 & 2.6005 & 2.60 \\
HSDT-8 & 3.9082 & 3.304 & 3.0083 & 2.7764 & 2.6599 & 1.04 \\
HSDT-9 & 3.8385 & 3.2467 & 3.0264 & 2.7124 & 2.5986 & \\
\hline
\end{tabular}

Where Avg. Diff $\%=$ Abs ((Present result- 3D result)/3D result $) \times 100$

Table 6: Comparison of non-dimensional frequency parameter with 3-D solution and other HSDTs of SS FGM-2 plate with different span to thickness ratio $(a=b=1, a / h=10)$

\begin{tabular}{ccccccc}
\hline Theories & \multicolumn{5}{c}{ Grading index } & \multirow{2}{*}{ Average Diff \% } \\
\cline { 2 - 5 } & 0 & 0.1 & 1 & 5 & 10 & \\
\hline 3D Solution [76] & 1.9339 & 1.8788 & 1.6583 & 1.5491 & 1.5066 & \\
IHHSDT & 1.9307 & 1.8775 & 1.6587 & 1.5494 & 1.5079 & 0.073 \\
HSDT-1 & 1.9307 & 1.8774 & 1.6588 & 1.5494 & 1.5079 & 0.075 \\
HSDT-2 & 1.9308 & 1.8776 & 1.6589 & 1.5494 & 1.508 & 0.075 \\
HSDT-3 & 1.9373 & 1.8836 & 1.6642 & 1.5576 & 1.5154 & 0.384 \\
HSDT-4 & 1.932 & 1.8788 & 1.6599 & 1.5505 & 1.509 & 0.089 \\
HSDT-5 & 1.932 & 1.8786 & 1.6597 & 1.551 & 1.5094 & 0.100 \\
HSDT-6 & 1.9312 & 1.878 & 1.6591 & 1.5497 & 1.5084 & 0.078 \\
HSDT-7 & 1.9422 & 1.8883 & 1.6685 & 1.5616 & 1.5199 & 0.648 \\
HSDT-8 & 1.9561 & 1.9012 & 1.6799 & 1.5764 & 1.5341 & 1.446 \\
HSDT-9 & 1.9368 & 1.8831 & 1.6638 & 1.557 & 1.5154 & 0.361 \\
HSDT-10 & 1.9319 & 1.8787 & 1.6597 & 1.5502 & 1.509 & 0.085 \\
HSDT-11 & 1.9311 & 1.8778 & 1.659 & 1.5499 & 1.5085 & 0.084 \\
HSDT-12 & 1.9392 & 1.8854 & 1.6658 & 1.5596 & 1.5173 & 0.493 \\
HSDT-13 & 1.9309 & 1.8769 & 1.6589 & 1.5494 & 1.5086 & 0.089 \\
HSDT-14 & 1.9317 & 1.8785 & 1.6595 & 1.55 & 1.5088 & 0.081 \\
\hline
\end{tabular}

ity. For checking the accuracy of the proposed theory, the numerical results for the uniaxial critical buckling parameter and non-dimensional frequency parameter are examined in Table 5 and Table 6, respectively. A SS FGM square plate is taken for investigation with different grading index. It can be observed from Table 5 and Table 6 that the proposed IHHSDT is very close to the 3-D exact solution results and also give low average Diff \% for the results obtained by other existing HSDT theories solved by the present method. So for further analysis, IHHSDT is considering for investigation.
Comparison study of present results with the other HSDT and FSDT result carried out with the different methods is listed in Table 7. Fundamental frequency parameter of FGM-1 plate for different values of span to thickness ratio $(\mathrm{a} / \mathrm{h}=5,10$, and 20$)$ is presented, with power index ' $\mathrm{n}$ ' $=0,0.5,1,4$, and 10 . It should be noted that the results reported by Hosseini-Hashemi et al., [78] has carried out an exact solution , Nguyen-Xuan et al., [79], Nguyen-Xuan et al., [80], Hosseini-Hashemi et al., [81], Zhao et al., [82] and Tran et al., [83] carried out FSDT with different values of shear correction factors in their study work and the results 
Table 7: Comparison of fundamental frequency parameter $\bar{\omega}=\omega h \sqrt{\rho_{c} / E_{c}}$ for FGM-1 square plates $(\mathrm{a} / \mathrm{b}=1)$

\begin{tabular}{ccccccc}
\hline $\mathrm{a} / \mathrm{h}$ & Theory & \multicolumn{5}{c}{ Grading index ' $\mathrm{n}$ ' } \\
\cline { 3 - 6 } & & 0 & 0.5 & 1 & 4 & 10 \\
\hline 5 & Ref. [78] & 0.2112 & 0.1805 & 0.1631 & 0.1397 & 0.1324 \\
& Ref. [79] & 0.2114 & 0.1805 & 0.163 & 0.1394 & 0.1323 \\
& Ref. [80] & 0.2127 & 0.1816 & 0.164 & 0.1403 & 0.1331 \\
& Ref. [84] & 0.2112 & 0.1806 & 0.1628 & 0.1375 & 0.13 \\
& Ref. [81] & 0.2112 & 0.1806 & 0.165 & 0.1371 & 0.1304 \\
& Ref. [82] & 0.2055 & 0.1757 & 0.1587 & 0.1356 & 0.1284 \\
& Ref. [83] & 0.214 & 0.1826 & 0.1649 & 0.1414 & 0.1343 \\
& Ref. [83] & 0.2113 & 0.1807 & 0.1631 & 0.1377 & 0.13 \\
& IHHST & 0.2096 & 0.1796 & 0.1622 & 0.1369 & 0.129 \\
& Ref. [78] & 0.0577 & 0.049 & 0.0442 & 0.0382 & 0.0366 \\
& Ref. [79] & 0.0578 & 0.0491 & 0.0443 & 0.0383 & 0.0366 \\
& Ref. [80] & 0.0582 & 0.0494 & 0.0445 & 0.0385 & 0.0368 \\
& Ref. [84] & 0.0577 & 0.049 & 0.0442 & 0.038 & 0.0364 \\
& Ref. [81] & 0.0578 & 0.0492 & 0.0443 & 0.0381 & 0.0364 \\
& Ref. [82] & 0.0567 & 0.0482 & 0.0435 & 0.0376 & 0.0359 \\
& Ref. [83] & 0.0579 & 0.0492 & 0.0443 & 0.0384 & 0.0367 \\
& Ref. [83] & 0.0577 & 0.049 & 0.0442 & 0.038 & 0.0363 \\
& IHHSDT & 0.0577 & 0.04905 & 0.0443 & 0.03781 & 0.0364 \\
& Ref. [78] & 0.0148 & 0.0125 & 0.0113 & 0.0098 & 0.0094 \\
& Ref. [79] & 0.0148 & 0.0126 & 0.0113 & 0.0098 & 0.0094 \\
& Ref. [80] & 0.0149 & 0.0127 & 0.0114 & 0.0099 & 0.0095 \\
& Ref. [84] & 0.0148 & 0.0125 & 0.0113 & 0.0098 & 0.0094 \\
Ref. [81] & 0.0148 & 0.0128 & 0.0115 & 0.0101 & 0.0096 \\
& Ref. [82] & 0.0146 & 0.0124 & 0.0112 & 0.0097 & 0.0093 \\
& Ref. [83] & 0.0148 & 0.0126 & 0.0113 & 0.0098 & 0.0094 \\
& Ref. [83] & 0.0148 & 0.0125 & 0.0113 & 0.0098 & 0.0094 \\
& IHHSDT & 0.01479 & 0.0125 & 0.0113 & 0.0098 & 0.0094 \\
\hline
\end{tabular}

reported by Benachour et al., [84], Tran et al., [83] has carried out HSDT. It can observed that present results are in good agreement with the other method result.

To verify the accuracy of the present method and theory for FGM plate on elastic foundation, buckling load parameter, and frequency parameter of the plates compared with those reported in the literature. Table 8 and Table 9 represent a comparison study on the effect of the elastic foundation for buckling load parameter and frequency parameter obtained by the present theory of SS FGM-1 plate, respectively. It can notice that the results of the present theory are in good agreement with those generated by TSDT, Thai and Kim, [85] and Thai and Choi, [86] for buckling load parameter and frequency parameter respectively. So, it can conclude that IHHSDT and method is not only accurate and stable but also efficient in critical buckling load parameter and frequency parameter of plates on elastic foundation.

\subsection{Parameter studies}

After verifying the accuracy and stability of the present method and proposed theory, parameter studies are carried out to examined the effects of grading index ' $n$ ', porosity distribution, porosity index span to thickness ratio, and foundation parameter on the buckling load and frequency parameter. After convergence and comparison studies of proposed theory and method, a $15 \times 15$ nodes and the proposed IHHSDT is considered for throughout the parameter studies considering the computational cost and accuracy of the studies.

Table 10 and Table 11 show the influences of porosity distribution and porosity index on critical buckling load parameter and critical natural frequency parameter of square SS porous FGM plate with various ' $n$ ' $=(0,1,2,5,8$, and 10) respectively. The materials property for Table 10 and Table 11 are FGM-4 and FGM-3 for a thick plate with 
Table 8: Comparison for the non-dimensional critical buckling load parameter $\bar{N}_{c r}=N_{c r} a^{2} / E h^{3}$ for the uniaxial SS FGM-1 plate on foundation $(\mathrm{a}=\mathrm{b}=1, \mathrm{FGM} 1, \mathrm{P}=0)$

\begin{tabular}{|c|c|c|c|c|c|c|c|c|}
\hline \multirow[t]{2}{*}{$(K w, K s)$} & \multirow[t]{2}{*}{$a / h$} & \multirow[t]{2}{*}{ Method } & \multicolumn{6}{|c|}{ Grading index ' $n$ ' } \\
\hline & & & 0 & 0.5 & 1 & 2 & 5 & 10 \\
\hline \multirow[t]{6}{*}{$(0,0)$} & 5 & Ref., [85] & 16.021 & 10.625 & 8.224 & 6.343 & 5.053 & 4.480 \\
\hline & & Present method & 16.173 & 10.736 & 8.327 & 6.438 & 5.129 & 4.541 \\
\hline & 10 & Ref., [85] & 18.579 & 12.123 & 9.339 & 7.263 & 6.035 & 5.452 \\
\hline & & Present method & 18.663 & 12.207 & 9.438 & 7.368 & 6.113 & 5.506 \\
\hline & 20 & Ref., [85] & 19.353 & 12.567 & 9.667 & 7.537 & 6.344 & 5.766 \\
\hline & & Present method & 19.403 & 12.644 & 9.778 & 7.664 & 6.437 & 5.821 \\
\hline \multirow[t]{6}{*}{$\left(10^{2}, 10\right)$} & 5 & Ref., [85] & 18.780 & 13.385 & 10.984 & 9.102 & 7.345 & 6.549 \\
\hline & & Present method & 18.937 & 13.498 & 11.088 & 9.197 & 7.446 & 6.629 \\
\hline & 10 & Ref., [85] & 21.338 & 14.882 & 12.099 & 10.022 & 8.794 & 8.212 \\
\hline & & Present method & 21.427 & 14.970 & 12.201 & 10.130 & 8.875 & 8.268 \\
\hline & 20 & Ref., [85] & 22.112 & 15.326 & 12.427 & 10.297 & 9.104 & 8.526 \\
\hline & & Present method & 22.166 & 15.407 & 12.541 & 10.426 & 9.199 & 8.584 \\
\hline \multirow[t]{6}{*}{$\left(10^{3}, 10^{2}\right)$} & 5 & Ref., [85] & 33.421 & 27.094 & 24.153 & 21.686 & 18.784 & 17.643 \\
\hline & & Present method & 33.634 & 27.248 & 24.289 & 21.801 & 18.880 & 17.713 \\
\hline & 10 & Ref., [85] & 40.648 & 31.461 & 27.432 & 24.347 & 22.36 & 21.452 \\
\hline & & Present method & 40.796 & 31.587 & 27.567 & 24.482 & 22.463 & 21.525 \\
\hline & 20 & Ref., [85] & 43.388 & 33.049 & 28.611 & 25.325 & 23.432 & 22.525 \\
\hline & & Present method & 43.476 & 33.152 & 28.742 & 25.468 & 23.539 & 22.592 \\
\hline
\end{tabular}

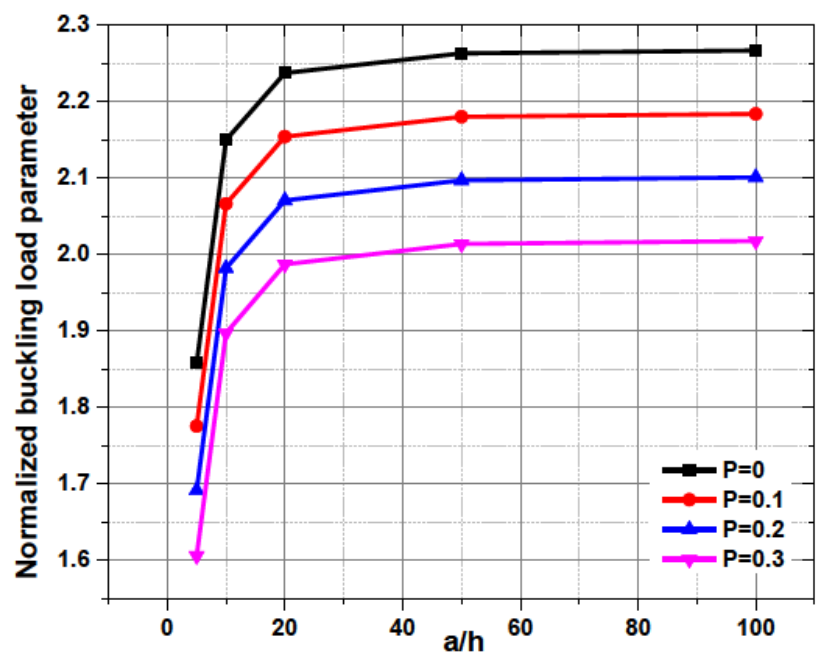

Figure 6: Effects of porosity index with various 'a/h' on critical buckling load parameter under biaxial loading of SS porous FGM-4 plate (SCPD, ‘n'=2,a=b)

$\mathrm{a} / \mathrm{h}=10$, respectively. It can be observed from both the tables that grading index moves from the ceramic layer to the metal layer, the critical buckling load parameter and critical natural frequency parameter start decreasing. The increase in ' $n$ ' transforms the pure ceramic into a combination of ceramic and metals phases. The combination increases the stiffness of the pure and porous FGM plate. Fur-

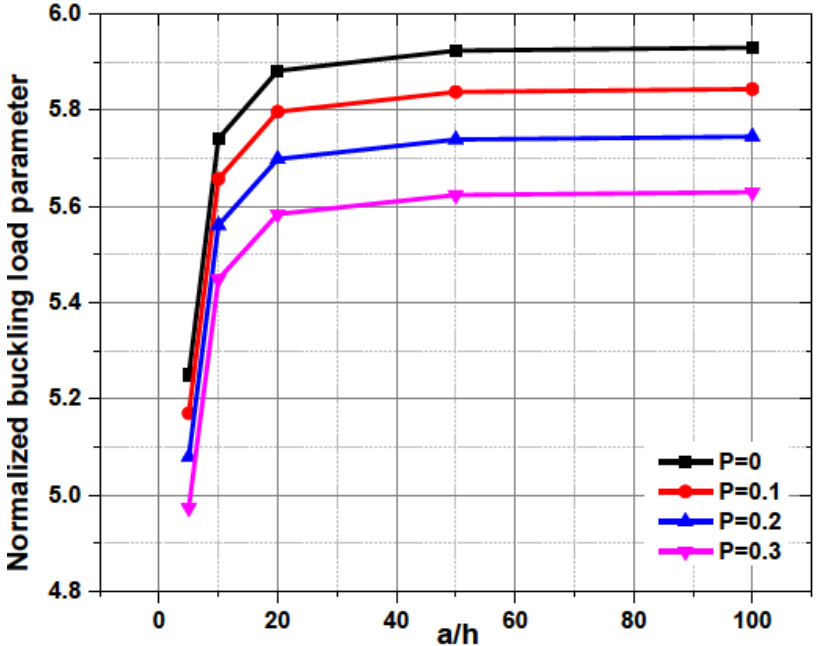

Figure 7: Effects of porosity index with various 'a/h' on critical natural frequency parameter of SS porous FGM-3 plate (SCPD, ' $n$ '=1,a=b)

ther, at $\mathrm{n}=0$, the plate is completely ceramics and obtained the highest critical buckling load parameter and critical natural frequency parameter. Three types of porosity distribution compared according to the porosity location. It can be observed that porosity denser in centers in SCPD predicts low effects as compared to porosity denser in top and bottom in STPD and SBPD distributions respectively. 
Table 9: Comparison of non-dimensional frequency parameter $\varpi=\left(\omega \sqrt{\rho_{m} / E_{m}}\right) / h$ of the SS FGM-1 plate $(\mathrm{a}=\mathrm{b}=1, \mathrm{FGM} 1, \mathrm{P}=0)$

\begin{tabular}{|c|c|c|c|c|c|c|c|c|c|}
\hline \multirow[t]{2}{*}{$\mathrm{Kw}$, } & \multirow[t]{2}{*}{ Ks } & \multirow[t]{2}{*}{$a / h$} & \multirow[t]{2}{*}{ Method } & \multicolumn{6}{|c|}{ Grading index ' $n$ ' } \\
\hline & & & & 0 & 0.5 & 1 & 2 & 5 & 10 \\
\hline \multirow[t]{6}{*}{0} & 0 & 5 & Ref. [86] & 10.376 & 8.876 & 8.012 & 7.231 & 6.668 & 6.388 \\
\hline & & & Present & 10.295 & 8.819 & 7.967 & 7.192 & 6.616 & 6.327 \\
\hline & & 10 & Ref. [86] & 11.335 & 9.630 & 8.682 & 7.876 & 7.403 & 7.145 \\
\hline & & & Present & 11.329 & 9.636 & 8.704 & 7.910 & 7.429 & 7.158 \\
\hline & & 20 & Ref. [86] & 11.631 & 9.859 & 8.886 & 8.075 & 7.639 & 7.392 \\
\hline & & & Present & 11.623 & 9.870 & 8.920 & 8.128 & 7.681 & 7.412 \\
\hline \multirow[t]{6}{*}{0} & 100 & 5 & Ref. [86] & 15.187 & 14.643 & 14.382 & 14.236 & 14.305 & 14.376 \\
\hline & & & Present & 15.036 & 14.507 & 14.246 & 14.091 & 14.332 & 14.405 \\
\hline & & 10 & Ref. [86] & 15.973 & 15.259 & 14.940 & 14.780 & 14.869 & 14.916 \\
\hline & & & Present & 15.948 & 15.238 & 14.926 & 14.769 & 14.850 & 14.889 \\
\hline & & 20 & Ref. [86] & 16.226 & 15.455 & 15.118 & 14.955 & 15.061 & 15.105 \\
\hline & & & Present & 16.206 & 15.445 & 15.120 & 14.966 & 15.063 & 15.095 \\
\hline \multirow[t]{6}{*}{100} & 0 & 5 & Ref. [86] & 10.672 & 9.256 & 8.452 & 7.741 & 7.253 & 7.018 \\
\hline & & & Present & 10.587 & 9.193 & 8.400 & 7.693 & 7.190 & 6.944 \\
\hline & & 10 & Ref. [86] & 11.615 & 9.992 & 9.104 & 8.364 & 7.952 & 7.729 \\
\hline & & & Present & 11.608 & 9.997 & 9.123 & 8.395 & 7.974 & 7.739 \\
\hline & & 20 & Ref. [86] & 11.906 & 10.216 & 9.303 & 8.558 & 8.179 & 7.964 \\
\hline & & & Present & 11.899 & 10.227 & 9.335 & 8.608 & 8.218 & 7.983 \\
\hline \multirow[t]{6}{*}{100} & 100 & 5 & Ref. [86] & 15.390 & 14.876 & 14.631 & 14.500 & 14.584 & 14.664 \\
\hline & & & Present & 15.238 & 14.737 & 14.492 & 14.127 & 14.352 & 14.425 \\
\hline & & 10 & Ref. [86] & 16.173 & 15.490 & 15.189 & 15.046 & 15.150 & 15.205 \\
\hline & & & Present & 16.148 & 15.469 & 15.174 & 15.034 & 15.130 & 15.177 \\
\hline & & 20 & Ref. [86] & 16.425 & 15.685 & 15.366 & 15.221 & 15.341 & 15.393 \\
\hline & & & Present & 16.405 & 15.676 & 15.369 & 15.232 & 15.344 & 15.383 \\
\hline
\end{tabular}

Table 10: Effects of porosity distribution with various ' $n$ ' on critical buckling load parameter $\bar{N}_{c r}=N_{c r} a^{2} / E_{c} h^{3}$ of SS porous FGM-4 plate uniaxial loading $(\mathrm{a} / \mathrm{h}=10, \mathrm{a}=\mathrm{b})$

\begin{tabular}{cccccccc}
\hline Porosity distribution & Porosity index 'P' & \multicolumn{7}{c}{ Grading index ' $\mathrm{n}$ ' } \\
\cline { 3 - 7 } & & 0 & 1 & 2 & 5 & 8 & 10 \\
\hline Pure FGM & 0 & 5.772 & 4.550 & 4.300 & 4.066 & 3.949 & 3.895 \\
SCPD & 0.1 & 5.547 & 4.371 & 4.133 & 3.911 & 3.800 & 3.748 \\
& 0.2 & 5.321 & 4.190 & 3.965 & 3.755 & 3.649 & 3.599 \\
& 0.3 & 5.094 & 4.007 & 3.795 & 3.597 & 3.497 & 3.450 \\
STPD & 0.1 & 5.429 & 4.277 & 4.042 & 3.824 & 3.717 & 3.666 \\
& 0.2 & 5.074 & 3.995 & 3.774 & 3.574 & 3.475 & 3.428 \\
& 0.3 & 4.705 & 3.701 & 3.495 & 3.312 & 3.223 & 3.180 \\
SBPD & 0.1 & 5.429 & 4.282 & 4.048 & 3.826 & 3.715 & 3.663 \\
& 0.2 & 5.074 & 4.004 & 3.787 & 3.577 & 3.472 & 3.423 \\
& 0.3 & 4.705 & 3.716 & 3.516 & 3.319 & 3.219 & 3.173 \\
\hline
\end{tabular}

It also noticed that by increasing the value of porosity index, critical buckling load parameter, and critical natural frequency parameter decreases.

Figure 6 and Figure 7 represent the influences of span to thickness ratio on critical buckling load parameter and critical natural frequency parameter with $\mathrm{n}=1$ for $\mathrm{SS}$ porous FGM plate. SCPD porosity distribution taken. It can be seen from both the tables that critical buckling load parameter and critical natural frequency parameter increase from thick to the thin plate for the porous FGM and the 
Table 11: Effects of porosity distribution with various ' $n$ ' on critical natural frequency parameter $\varpi=\left(\omega \sqrt{\rho_{m} / E_{m}}\right) / h$ of SS porous FGM-3 plate $(a / h=10, a=b)$

\begin{tabular}{cccccccc}
\hline Porosity distribution & Porosity index 'P' & \multicolumn{7}{c}{ Grading index ' $\mathrm{n}$ ' } \\
\cline { 3 - 7 } & & 0 & 1 & 2 & 5 & 8 & 10 \\
\hline Pure FGM & 0 & 7.628 & 5.741 & 5.286 & 4.930 & 4.777 & 4.704 \\
SCPD & 0.1 & 7.599 & 5.713 & 5.264 & 4.915 & 4.764 & 4.693 \\
& 0.2 & 7.568 & 5.682 & 5.240 & 4.897 & 4.750 & 4.679 \\
& 0.3 & 7.533 & 5.647 & 5.212 & 4.877 & 4.732 & 4.663 \\
STPD & 0.1 & 7.518 & 5.658 & 5.207 & 4.857 & 4.709 & 4.639 \\
& 0.2 & 7.391 & 5.561 & 5.114 & 4.771 & 4.629 & 4.561 \\
& 0.3 & 7.240 & 5.446 & 5.005 & 4.669 & 4.533 & 4.468 \\
SBPD & 0.1 & 7.518 & 5.657 & 5.213 & 4.863 & 4.710 & 4.637 \\
& 0.2 & 7.391 & 5.561 & 5.129 & 4.784 & 4.632 & 4.559 \\
& 0.3 & 7.240 & 5.449 & 5.031 & 4.692 & 4.539 & 4.467 \\
\hline
\end{tabular}

Table 12: Effects of foundation and porosity index on critical buckling load parameter and critical natural frequency parameter of SS porous FGM-3 plate (SBPD, ‘a/h'=10, $a=b)$

\begin{tabular}{|c|c|c|c|c|c|c|c|c|c|c|c|c|}
\hline \multirow[t]{3}{*}{ Ks } & \multirow[t]{3}{*}{$\mathrm{Kw}$} & \multirow[t]{3}{*}{ ‘P’ } & \multicolumn{10}{|c|}{ Grading index ' $n$ ' } \\
\hline & & & \multicolumn{5}{|c|}{$\bar{N}_{c r}=N_{c r} a^{2} / E_{c} h^{3}$} & \multicolumn{5}{|c|}{$\varpi=\left(\omega \sqrt{\rho_{m} / E_{m}}\right) / h$} \\
\hline & & & 0 & 1 & 2 & 5 & 10 & 0 & 1 & 2 & 5 & 10 \\
\hline \multirow[t]{3}{*}{0} & 0 & 0 & 10.19 & 6.30 & 5.49 & 4.90 & 4.51 & 7.63 & 5.74 & 5.29 & 4.93 & 4.70 \\
\hline & & 0.1 & 9.58 & 5.93 & 5.17 & 4.62 & 4.25 & 7.60 & 5.71 & 5.26 & 4.91 & 4.69 \\
\hline & & 0.3 & 8.30 & 5.14 & 4.50 & 4.02 & 3.68 & 7.53 & 5.65 & 5.21 & 4.88 & 4.66 \\
\hline \multirow[t]{3}{*}{0} & 10 & 0 & 10.28 & 6.39 & 5.58 & 4.99 & 4.61 & 7.66 & 5.78 & 5.33 & 4.98 & 4.75 \\
\hline & & 0.1 & 9.67 & 6.02 & 5.26 & 4.71 & 4.34 & 7.55 & 5.70 & 5.26 & 4.91 & 4.69 \\
\hline & & 0.3 & 8.40 & 5.24 & 4.60 & 4.11 & 3.78 & 7.28 & 5.50 & 5.08 & 4.75 & 4.52 \\
\hline \multirow[t]{3}{*}{0} & 100 & 0 & 11.12 & 7.23 & 6.42 & 5.83 & 5.44 & 7.97 & 6.15 & 5.72 & 5.38 & 5.17 \\
\hline & & 0.1 & 10.51 & 6.86 & 6.10 & 5.55 & 5.18 & 7.87 & 6.09 & 5.66 & 5.33 & 5.12 \\
\hline & & 0.3 & 9.23 & 5.43 & 5.43 & 6.07 & 4.61 & 7.64 & 5.92 & 5.53 & 5.21 & 5.00 \\
\hline \multirow[t]{3}{*}{10} & 0 & 0 & 12.02 & 8.13 & 7.32 & 6.73 & 6.34 & 8.28 & 6.52 & 6.10 & 5.78 & 5.58 \\
\hline & & 0.1 & 11.41 & 7.75 & 7.00 & 6.45 & 6.08 & 8.20 & 6.47 & 6.07 & 5.75 & 5.55 \\
\hline & & 0.3 & 10.13 & 6.97 & 6.33 & 5.85 & 5.51 & 8.00 & 6.34 & 5.97 & 5.66 & 5.46 \\
\hline \multirow[t]{3}{*}{100} & 0 & 0 & 26.24 & 20.62 & 19.39 & 18.44 & 17.87 & 12.75 & 11.34 & 11.00 & 10.73 & 10.58 \\
\hline & & 0.1 & 25.35 & 20.07 & 18.92 & 18.02 & 17.48 & 12.82 & 11.44 & 11.11 & 10.83 & 10.68 \\
\hline & & 0.3 & 23.49 & 18.92 & 17.94 & 17.16 & 16.67 & 12.96 & 11.63 & 11.32 & 11.06 & 10.91 \\
\hline \multirow[t]{3}{*}{100} & 100 & 0 & 26.47 & 20.85 & 19.62 & 18.67 & 18.10 & 12.96 & 11.56 & 11.22 & 10.94 & 10.79 \\
\hline & & 0.1 & 25.59 & 20.30 & 19.15 & 18.26 & 17.71 & 13.04 & 11.66 & 11.32 & 11.05 & 10.90 \\
\hline & & 0.3 & 23.72 & 19.15 & 18.17 & 17.39 & 16.90 & 13.18 & 11.86 & 11.55 & 11.28 & 13.18 \\
\hline
\end{tabular}

entire porosity index follow the same trend. The effect of thick to the thin plate is negligible after $\mathrm{a} / \mathrm{h}=50$. Table 12 represents the influences of foundation parameters with a porosity index on the critical buckling load parameter and critical natural frequency parameter of SS porous FGM-3 plate. SBPD porosity distribution and span to thickness ratio considered. It can see that by increasing the value of Winkler and Pasternak parameters cause to increase in the buckling load and natural frequency. The table shows also, Pasternak parameter of the foundation has a more significant effect than Winkler parameter on the buckling load and fundamental frequency of plate.

Table 13 represents the effect of porosity index on the non-dimensional buckling load parameter for eight modes. SCPD porosity distribution is taken with $n=1$ and $a / h=10$. It can be seen that by increasing the porosity index, non- 
Table 13: Non-dimensional buckling load parameter $\bar{N}=N a^{2} / E_{m} h^{3}$ of eight modes for SS porous FGM-1 plate on foundation (a=b=1, FGM $1, n=1, a / h=10$, SCPD)

\begin{tabular}{lccccccccc}
\hline Modes & \multicolumn{3}{c}{ Uniaxial loading } & \multicolumn{3}{c}{ Biaxial loading } & \multicolumn{3}{c}{ Pure shear loading } \\
\cline { 2 - 10 } & 0 & 0.1 & 0.3 & 0 & 0.1 & 0.3 & 0 & 0.1 & 0.3 \\
\hline Mode 1 & 9.438 & 9.027 & 7.681 & 4.719 & 4.434 & 3.840 & 19.949 & 18.725 & 16.183 \\
Mode 2 & 13.805 & 13.172 & 11.207 & 11.050 & 10.375 & 8.971 & 19.949 & 18.725 & 16.183 \\
Mode 3 & 22.148 & 21.062 & 17.906 & 11.050 & 10.375 & 8.971 & 23.778 & 22.309 & 19.265 \\
Mode 4 & 31.719 & 30.054 & 25.515 & 16.787 & 15.749 & 13.597 & 23.778 & 22.309 & 19.265 \\
Mode 5 & 33.556 & 31.950 & 27.185 & 19.916 & 18.674 & 16.104 & 41.738 & 39.060 & 33.546 \\
Mode 6 & 35.880 & 34.063 & 28.955 & 19.959 & 18.713 & 16.135 & 41.738 & 39.060 & 33.546 \\
Mode 7 & 41.191 & 38.893 & 32.971 & 24.849 & 23.284 & 20.052 & 43.814 & 40.990 & 35.183 \\
Mode 8 & 42.417 & 40.138 & 34.073 & 24.849 & 23.284 & 20.052 & 43.814 & 40.990 & 35.183 \\
\hline
\end{tabular}

Table 14: Non-dimensional critical buckling load parameter $\bar{N}_{c r}=N_{c r} a^{2} / E_{m} h^{3}$ for different type of in-plane loading SS porous FGM-1 plate on foundation ( $a=b=1, F G M 2, a / h=10, S C P D)$

\begin{tabular}{cccccccc}
\hline $\mathrm{n}$ & $\mathrm{P}$ & Uniaxial & Uniaxial + Shear & Diff\% & Biaxial & Biaxial + Shear & Diff\% \\
\hline 0 & 0 & 7.416 & 6.238 & 15.88 & 3.708 & 3.522 & 5.00 \\
& 0.1 & 7.126 & 5.987 & 15.98 & 3.563 & 3.383 & 5.04 \\
& 0.3 & 6.541 & 5.478 & 16.25 & 3.270 & 3.102 & 5.14 \\
1 & 0 & 5.204 & 4.382 & 15.80 & 2.602 & 2.473 & 4.97 \\
& 0.1 & 4.995 & 4.201 & 15.90 & 2.497 & 2.372 & 5.00 \\
& 0.3 & 4.572 & 3.833 & 16.15 & 2.286 & 2.169 & 5.10 \\
2 & 0 & 4.755 & 3.996 & 15.97 & 2.377 & 2.258 & 5.03 \\
& 0.1 & 4.569 & 3.834 & 16.07 & 2.284 & 2.168 & 5.07 \\
& 0.3 & 4.191 & 3.506 & 16.35 & 2.095 & 1.987 & 5.18 \\
5 & 0 & 4.384 & 3.672 & 16.22 & 2.192 & 2.079 & 5.13 \\
& 0.1 & 4.217 & 3.528 & 16.34 & 2.109 & 1.999 & 5.17 \\
& 0.3 & 3.880 & 3.234 & 16.65 & 1.940 & 1.837 & 5.29 \\
\hline
\end{tabular}

Table 15: Effects of porosity index with various ‘ $a / h$ ' on natural frequency parameter of four modes of SS porous FGM-2 plate (SCPD, ' $n$ '=1,a=b)

\begin{tabular}{|c|c|c|c|c|c|c|c|c|c|}
\hline \multirow[t]{2}{*}{ Modes } & \multicolumn{3}{|c|}{$a / h=5$} & \multicolumn{3}{|c|}{$a / h=10$} & \multicolumn{3}{|c|}{$a / h=20$} \\
\hline & $\mathrm{P}=0$ & $\mathrm{P}=0.1$ & $\mathrm{P}=0.3$ & $\mathrm{P}=0$ & $\mathrm{P}=0.1$ & $\mathrm{P}=0.3$ & $\mathrm{P}=0$ & $\mathrm{P}=0.1$ & $\mathrm{P}=0.3$ \\
\hline Mode 1 & 6.05 & 6.01 & 5.90 & 6.64 & 6.61 & 6.55 & 6.81 & 6.79 & 6.73 \\
\hline Mode 2 & 10.88 & 10.70 & 10.29 & 15.82 & 15.73 & 15.50 & 16.83 & 16.76 & 16.61 \\
\hline Mode 3 & 10.88 & 10.70 & 10.29 & 15.84 & 15.74 & 15.51 & 16.83 & 16.76 & 16.61 \\
\hline Mode 4 & 12.74 & 12.59 & 12.21 & 22.03 & 21.66 & 20.83 & 26.68 & 26.56 & 26.29 \\
\hline
\end{tabular}

dimensional buckling load parameter starts decreasing in all loading condition. By increasing the mode numbers, non-dimensional buckling load parameter also increase in all three loading conditions. It also noticed that pure shear loading is more dominating than other loadings. Table 14 represents the effect of loading with and without the shear effect of SS porous FGM-2 plate. SCPD porosity distribution is taken with $\mathrm{a} / \mathrm{h}=10$. It can be seen that Diff $\%$ of uniaxial loading with and without shear on the non-dimensional critical buckling load parameter is more dominating as compare to the Diff \% of biaxial loading with and without shear, this indicates that shear loading is more dominating with uniaxial loading as compared to the biaxial loading. Non-dimensional critical buckling load parameter starts decreasing with increasing porosity index in all the inplane loadings. Table 15 represents the effects of porosity index on natural frequency parameter of four modes of SS porous FGM-2 plate. It can be seen that natural frequency 
parameter starts decreasing by increasing the porosity index in all modes and by increasing the spam to thickness ratios the natural frequency parameter increasing.

\section{Concluding remarks}

In this work, a new five variables inverse hyperbolic higherorder shear deformation theory (IHHSDT) developed for the buckling and free vibration of porous FGM plates resting on two-parameter elastic foundations. The new nonpolynomial IHHSDT with five variables developed and satisfied zero transverse shear stress boundary condition at the top and bottom surfaces of the plate. Therefore, a shear correction factor is not required. The accuracy and efficacy of the present theory verified by comparing it with many existing results in the literature. It noticed that the developed theory is closer to the three-dimensional results and better than the results of the existing theories. The results obtained by the proposed theory and the present method can be summarized as follows:

- It has been shown that the present MQ-RBF based meshfree method can accurately predict buckling load and natural frequencies of FGM plate with and without resting on an elastic foundation.

- The buckling loads and natural frequencies of plate decrease with the increase of power index.

- The buckling loads and natural frequencies increase from thick to a thin plate

- The buckling loads and natural frequencies of plate decrease with the increase of porosity index.

- The shear loading is more dominating with uniaxial loading as compared to the biaxial loading for buckling loads

- Porosity distribution located at the center (SCPD) predict buckling loads and natural frequencies closer to the pure FGM plate as compared to the STPD and SBPD

- The buckling loads and natural frequencies increase by increasing the value of Winkler and Pasternak parameters.

- Pasternak elastic foundation has a more significant rule as compared to Winkler elastic foundation in increasing the buckling load and natural frequency.

The newly developed theory and the current method can recommended as more correct and efficient for analyzing the buckling and free vibration behavior of porous FGM plate. The reported results will serve as a benchmark numerical solution to validate other solution methodolo- gies and theory will be useful to the research community working in this field and in FGM applications.

\section{References}

[1] M.B. Bever, P.E. Duwez, Gradients in composite materials, Materials Science and Engineering. 10 (1972) 1-8. doi:10.1016/00255416(72)90059-6.

[2] Levy M, Memoire sur la theorie des plaques elastiques planes, J Math Pures Appl. 30 (1877) 219-306.

[3] M. Touratier, An efficient standard plate theory, International Journal of Engineering Science. 29 (1991) 901-916. doi:10.1016/0020-7225(91)90165-Y.

[4] K.P. Soldatos, A transverse shear deformation theory for homogeneous monoclinic plates, Acta Mechanica. 94 (1992) 195-220. doi:10.1007/BF01176650.

[5] M. Karama, K.S. Afaq, S. Mistou, A new theory for laminated composite plates, Proceedings of the IMechE. 223 (2009) 53-62. doi:10.1243/14644207JMDA189.

[6] J.L. Mantari, A.S. Oktem, C. Guedes Soares, A new higher order shear deformation theory for sandwich and composite laminated plates, Composites Part B: Engineering. 43 (2012) 1489-1499. doi:10.1016/j.compositesb.2011.07.017.

[7] N. Grover, D.K. Maiti, B.N. Singh, A new inverse hyperbolic shear deformation theory for static and buckling analysis of laminated composite and sandwich plates, Composite Structures. 95 (2013) 667-675. doi:10.1016/j.compstruct.2012.08.012.

[8] J.L. Mantari, C. Guedes Soares, Static response of advanced composite plates by a new non-polynomial higher-order shear deformation theory, International Journal of Mechanical Sciences. 78 (2014) 60-71. doi:10.1016/j.ijmecsci.2013.10.020.

[9] Aicha Bessaim, Mohammed SA Houari, Abdelouahed Tounsi, SR Mahmoud, El Abbes Adda Bedia, A new higher-order shear and normal deformation theory for the static and free vibration analysis of sandwich plates with functionally graded isotropic face sheets, Jnl of Sandwich Structures \& Materials. 15 (2013) 671-703. doi:10.1177/1099636213498888.

[10] N. El Meiche, A. Tounsi, N. Ziane, I. Mechab, E.A. Adda.Bedia, A new hyperbolic shear deformation theory for buckling and vibration of functionally graded sandwich plate, International Journal of Mechanical Sciences. 53 (2011) 237-247. doi:10.1016/j.ijmecsci.2011.01.004.

[11] H.-T. Thai, T.P. Vo, A new sinusoidal shear deformation theory for bending, buckling, and vibration of functionally graded plates, Applied Mathematical Modelling. 37 (2013) 3269-3281. doi:10.1016/j.apm.2012.08.008.

[12] A. Mahi, E.A. Adda Bedia, A. Tounsi, A new hyperbolic shear deformation theory for bending and free vibration analysis of isotropic, functionally graded, sandwich and laminated composite plates, Applied Mathematical Modelling. 39 (2015) 24892508. doi:10.1016/j.apm.2014.10.045.

[13] Y.S. Joshan, N. Grover, B.N. Singh, A new non-polynomial four variable shear deformation theory in axiomatic formulation for hygro-thermo-mechanical analysis of laminated composite plates, Composite Structures. 182 (2017) 685-693. doi:10.1016/j.compstruct.2017.09.029. 
[14] M. Aydogdu, A new shear deformation theory for laminated composite plates, Composite Structures. 89 (2009) 94-101. doi:10.1016/j.compstruct.2008.07.008.

[15] J.L. Mantari, Computational Development of a 4-Unknowns Trigonometric Quasi-3D Shear Deformation Theory to Study Advanced Sandwich Plates and Shells, Int. J. Appl. Mechanics. 08 (2016) 1650049. doi:10.1142/S1758825116500496.

[16] E. Viola, F. Tornabene, N. Fantuzzi, Static analysis of completely doubly-curved laminated shells and panels using general higherorder shear deformation theories, Composite Structures. 101 (2013) 59-93. doi:10.1016/j.compstruct.2013.01.002.

[17] E. Viola, F. Tornabene, N. Fantuzzi, General higherorder shear deformation theories for the free vibration analysis of completely doubly-curved laminated shells and panels, Composite Structures. 95 (2013) 639-666. doi:10.1016/j.compstruct.2012.08.005.

[18] B. Nayroles, G. Touzot, P. Villon, Generalizing the finite element method: Diffuse approximation and diffuse elements, Computational Mechanics. 10 (1992) 307-318. doi:10.1007/BF00364252.

[19] Multiquadric equations of topography and other irregular surfaces, Journal of Geophysical Research. 76 (1971) 1905-1915. doi:10.1029/JB076i008p01905.

[20] E.J. Kansa, Multiquadrics-A scattered data approximation scheme with applications to computational fluid-dynamics-I surface approximations and partial derivative estimates, Computers \& Mathematics with Applications. 19 (1990) 127-145. doi:10.1016/0898-1221(90)90270-T.

[21] R. Franke, Smooth interpolation of scattered data by local thin plate splines, Computers \& Mathematics with Applications. 8 (1982) 273-281. doi:10.1016/0898-1221(82)90009-8.

[22] A.H.-D. Cheng, Multiquadric and its shape parameter-A numerical investigation of error estimate, condition number, and round-off error by arbitrary precision computation, Engineering Analysis with Boundary Elements. 36 (2012) 220-239. doi:10.1016/j.enganabound.2011.07.008.

[23] G.R. Liu, Y.T. Gu, A LOCAL RADIAL POINT INTERPOLATION METHOD (LRPIM) FOR FREE VIBRATION ANALYSES OF 2-D SOLIDS, Journal of Sound and Vibration. 246 (2001) 29-46. doi:10.1006/jsvi.2000.3626.

[24] G.R. Liu, Y.T. Gu, A point interpolation method for twodimensional solids, International Journal for Numerical Methods in Engineering. 50 (2001) 937-951. doi:10.1002/10970207(20010210)50:4<937::AID-NME62>3.0.CO;2-X.

[25] S.S. Chen, C.J. Xu, G.S. Tong, X. Wei, Free vibration of moderately thick functionally graded plates by a meshless local natural neighbor interpolation method, Engineering Analysis with Boundary Elements. 61 (2015) 114-126. doi:10.1016/j.enganabound.2015.07.008.

[26] R. Kumar, J. Singh, Assessment of higher order transverse shear deformation theories for modeling and buckling analysis of FGM plates using RBF based meshless approach, Multi Modelg in Mat \& Struct. (2018). doi:10.1108/MMMS-07-2017-0069.

[27] A.J.M. Ferreira, C.M.C. Roque, P.A.L.S. Martins, Analysis of composite plates using higher-order shear deformation theory and a finite point formulation based on the multiquadric radial basis function method, Composites Part B: Engineering. 34 (2003) 627-636. doi:10.1016/S1359-8368(03)00083-0.

[28] R. Kumar, A. Lal, B.N. Singh, J. Singh, New transverse shear deformation theory for bending analysis of $\mathrm{fgm}$ plate under patch load, Composite Structures. (2018). doi:10.1016/j.compstruct.2018.10.014.

[29] A.J.M. Ferreira, C.M.C. Roque, R.M.N. Jorge, G.E. Fasshauer, R.C. Batra, Analysis of Functionally Graded Plates by a Robust Meshless Method, Mechanics of Advanced Materials and Structures. 14 (2007) 577-587. doi:10.1080/15376490701672732.

[30] A.M.A. Neves, T.A. Driscoll, A.R.H. Heryudono, A.J.M. Ferreira, C.M.M. Soares, R.M.N. Jorge, Adaptive Methods for Analysis of Composite Plates with Radial Basis Functions, Mechanics of Advanced Materials and Structures. 18 (2011) 420-430. doi:10.1080/15376494.2010.528155.

[31] C.M.C. Roque, J.D. Rodrigues, A.J.M. Ferreira, Static Deformations and Vibration Analysis of Composite and Sandwich Plates Using a Layerwise Theory and a Local Radial Basis Functions-Finite Differences Discretization, Mechanics of Advanced Materials and Structures. 20 (2013) 666-678. doi:10.1080/15376494.2011.646053.

[32] A.J.M. Ferreira, C.M.C. Roque, E. Carrera, M. Cinefra, O. Polit, Bending and Vibration of Laminated Plates by a Layerwise Formulation and Collocation with Radial Basis Functions, Mechanics of Advanced Materials and Structures. 20 (2013) 624-637. doi:10.1080/15376494.2011.643282.

[33] J. Singh, S. Singh, K.K. Shukla, Meshless Analysis of Laminated Composite and Sandwich Plates Subjected to Various Types of Loads, International Journal for Computational Methods in Engineering Science and Mechanics. 15 (2014) 158-171. doi:10.1080/15502287.2013.874060.

[34] N. Fantuzzi, M. Bacciocchi, F. Tornabene, E. Viola, A.J.M. Ferreira, Radial basis functions based on differential quadrature method for the free vibration analysis of laminated composite arbitrarily shaped plates, Composites Part B: Engineering. 78 (2015) 65-78. doi:10.1016/j.compositesb.2015.03.027.

[35] P. Zhu, K.M. Liew, Free vibration analysis of moderately thick functionally graded plates by local Kriging meshless method, Composite Structures. 93 (2011) 2925-2944. doi:10.1016/j.compstruct.2011.05.011.

[36] A.J.M. Ferreira, R.C. Batra, C.M.C. Roque, L.F. Qian, R.M.N. Jorge, Natural frequencies of functionally graded plates by a meshless method, Composite Structures. 75 (2006) 593-600. doi:10.1016/j.compstruct.2006.04.018.

[37] W. Xiang, Y. Xing, A New First-Order Shear Deformation Theory for Free Vibrations of Rectangular Plate, Int. J. Appl. Mechanics. 07 (2014) 1550008. doi:10.1142/S1758825115400086.

[38] X. Zhao, Y.Y. Lee, K.M. Liew, Free vibration analysis of functionally graded plates using the element-free kp-Ritz method, Journal of Sound and Vibration. 319 (2009) 918-939. doi:10.1016/j.jsv.2008.06.025.

[39] T.-V. Vu, N.-H. Nguyen, A. Khosravifard, M.R. Hematiyan, S. Tanaka, T.Q. Bui, A simple FSDT-based meshfree method for analysis of functionally graded plates, Engineering Analysis with Boundary Elements. 79 (2017) 1-12. doi:10.1016/j.enganabound.2017.03.002.

[40] A.M.A. Neves, A.J.M. Ferreira, E. Carrera, M. Cinefra, C.M.C. Roque, R.M.N. Jorge, C.M.M. Soares, A quasi-3D hyperbolic shear deformation theory for the static and free vibration analysis of functionally graded plates, Composite Structures. 94 (2012) 1814-1825. doi:10.1016/j.compstruct.2011.12.005.

[41] C.-P. Wu, K.-H. Chiu, RMVT-based meshless collocation and element-free Galerkin methods for the quasi-3D free vibration analysis of multilayered composite and FGM plates, Composite Structures. 93 (2011) 1433-1448. 
doi:10.1016/j.compstruct.2010.11.015

[42] H.-T. Thai, D.-H. Choi, An efficient and simple refined theory for buckling analysis of functionally graded plates, Applied Mathematical Modelling. 36 (2012) 1008-1022. doi:10.1016/j.apm.2011.07.062.

[43] M. Park, D.-H. Choi, A two-variable first-order shear deformation theory considering in-plane rotation for bending, buckling and free vibration analyses of isotropic plates, Applied Mathematical Modelling. 61 (2018) 49-71. doi:10.1016/j.apm.2018.03.036.

[44] T.Q. Bui, M.N. Nguyen, Ch. Zhang, Buckling analysis of Reissner-Mindlin plates subjected to in-plane edge loads using a shear-locking-free and meshfree method, Engineering Analysis with Boundary Elements. 35 (2011) 1038-1053. doi:10.1016/j.enganabound.2011.04.001.

[45] S. Sadamoto, S. Tanaka, K. Taniguchi, M. Ozdemir, T.Q. Bui, C. Murakami, D. Yanagihara, Buckling analysis of stiffened plate structures by an improved meshfree flat shell formulation, Thin-Walled Structures. 117 (2017) 303-313. doi:10.1016/j.tws.2017.04.012.

[46] X. Zhao, Y.Y. Lee, K.M. Liew, Mechanical and thermal buckling analysis of functionally graded plates, Composite Structures. 90 (2009) 161-171. doi:10.1016/j.compstruct.2009.03.005.

[47] A. Moslemi, B. Navayi Neya, J. Vaseghi Amiri, Benchmark solution for buckling of thick rectangular transversely isotropic plates under biaxial load, International Journal of Mechanical Sciences. 131-132 (2017) 356-367. doi:10.1016/j.ijmecsci.2017.07.006.

[48] T. Yu, S. Yin, T.Q. Bui, C. Liu, N. Wattanasakulpong, Buckling isogeometric analysis of functionally graded plates under combined thermal and mechanical loads, Composite Structures. 162 (2017) 54-69. doi:10.1016/j.compstruct.2016.11.084.

[49] S. Chakraverty, K.K. Pradhan, Free vibration of functionally graded thin rectangular plates resting on winkler elastic foundation with general boundary conditions using rayleighritz method, Int. J. Appl. Mechanics. 06 (2014) 1450043. doi:10.1142/S1758825114500434.

[50] M. Talha, B.N. Singh, Static response and free vibration analysis of FGM plates using higher order shear deformation theory, Applied Mathematical Modelling. 34 (2010) 3991-4011. doi:10.1016/j.apm.2010.03.034.

[51] P. Malekzadeh, Three-dimensional free vibration analysis of thick functionally graded plates on elastic foundations, Composite Structures. 89 (2009) 367-373. doi:10.1016/j.compstruct.2008.08.007.

[52] H. Ait Atmane, A. Tounsi, I. Mechab, E.A. Adda Bedia, Free vibration analysis of functionally graded plates resting on WinklerPasternak elastic foundations using a new shear deformation theory, Int J Mech Mater Des. 6 (2010) 113-121. doi:10.1007/s10999. 010-9110-x.

[53] M. Sobhy, Buckling and free vibration of exponentially graded sandwich plates resting on elastic foundations under various boundary conditions, Composite Structures. 99 (2013) 76-87. doi:10.1016/j.compstruct.2012.11.018.

[54] D. Shahsavari, M. Shahsavari, L. Li, B. Karami, A novel quasi-3D hyperbolic theory for free vibration of FG plates with porosities resting on Winkler/Pasternak/Kerr foundation, Aerospace Science and Technology. 72 (2018) 134-149. doi:10.1016/j.ast.2017.11.004.

[55] Y.Q. Wang, Y.H. Wan, Y.F. Zhang, Vibrations of longitudinally traveling functionally graded material plates with porosities, European Journal of Mechanics - A/Solids. 66 (2017) 55-68. doi:10.1016/j.euromechsol.2017.06.006.

[56] J. Zhao, K. Choe, F. Xie, A. Wang, C. Shuai, Q. Wang, Threedimensional exact solution for vibration analysis of thick functionally graded porous (FGP) rectangular plates with arbitrary boundary conditions, Composites Part B: Engineering. 155 (2018) 369-381. doi:10.1016/j.compositesb.2018.09.001.

[57] M.C. Kiran, S.C. Kattimani, Assessment of porosity influence on vibration and static behaviour of functionally graded magneto-electro-elastic plate: A finite element study, European Journal of Mechanics - A/Solids. 71 (2018) 258-277. doi:10.1016/j.euromechsol.2018.04.006.

[58] J. Kim, K.K. Żur, J.N. Reddy, Bending, free vibration, and buckling of modified couples stress-based functionally graded porous micro-plates, Composite Structures. 209 (2019) 879-888. doi:10.1016/j.compstruct.2018.11.023.

[59] J.N. Reddy, A Simple Higher-Order Theory for Laminated Composite Plates, J. Appl. Mech. 51 (1984) 745-752. doi:10.1115/1.3167719.

[60] M. Touratier, An efficient standard plate theory, International Journal of Engineering Science. 29 (1991) 901-916. doi:10.1016/0020-7225(91)90165-Y.

[61] A.M. Zenkour, Generalized shear deformation theory for bending analysis of functionally graded plates, Applied Mathematical Modelling. 30 (2006) 67-84. doi:10.1016/j.apm.2005.03.009.

[62] J.L. Mantari, A.S. Oktem, C. Guedes Soares, Bending response of functionally graded plates by using a new higher order shear deformation theory, Composite Structures. 94 (2012) 714-723. doi:10.1016/j.compstruct.2011.09.007.

[63] H. Arya, R.P. Shimpi, N.K. Naik, A zigzag model for laminated composite beams, Composite Structures. 56 (2002) 21-24. doi:10.1016/S0263-8223(01)00178-7.

[64] M. Karama, K.S. Afaq, S. Mistou, A new theory for laminated composite plates, Proceedings of the IMechE. 223 (2009) 53-62. doi:10.1243/14644207JMDA189.

[65] M. Aydogdu, A new shear deformation theory for laminated composite plates, Composite Structures. 89 (2009) 94-101. doi:10.1016/j.compstruct.2008.07.008.

[66] N. Grover, D.K. Maiti, B.N. Singh, A new inverse hyperbolic shear deformation theory for static and buckling analysis of laminated composite and sandwich plates, Composite Structures. 95 (2013) 667-675. doi:10.1016/j.compstruct.2012.08.012.

[67] C.H. Thai, A.J.M. Ferreira, S.P.A. Bordas, T. Rabczuk, H. NguyenXuan, Isogeometric analysis of laminated composite and sandwich plates using a new inverse trigonometric shear deformation theory, European Journal of Mechanics - A/Solids. 43 (2014) 89108. doi:10.1016/j.euromechsol.2013.09.001.

[68] H. Nguyen-Xuan, C.H. Thai, T. Nguyen-Thoi, Isogeometric finite element analysis of composite sandwich plates using a higher order shear deformation theory, Composites Part B: Engineering. 55 (2013) 558-574. doi:10.1016/j.compositesb.2013.06.044.

[69] A. Mahi, E.A. Adda Bedia, A. Tounsi, A new hyperbolic shear deformation theory for bending and free vibration analysis of isotropic, functionally graded, sandwich and laminated composite plates, Applied Mathematical Modelling. 39 (2015) 24892508. doi:10.1016/j.apm.2014.10.045.

[70] D.B. Singh, B.N. Singh, New higher order shear deformation theories for free vibration and buckling analysis of laminated and braided composite plates, International Journal of Mechanical Sciences. 131-132 (2017) 265-277. doi:10.1016/j.ijmecsci.2017.06.053. 
[71] T.N. Nguyen, C.H. Thai, H. Nguyen-Xuan, On the general framework of high order shear deformation theories for laminated composite plate structures: A novel unified approach, International Journal of Mechanical Sciences. 110 (2016) 242-255. doi:10.1016/j.ijmecsci.2016.01.012.

[72] An efficient shear deformation theory for free vibration of functionally graded thick rectangular plates on elastic foundation, Composite Structures. 108 (2014) 667-676. doi:10.1016/j.compstruct.2013.10.019.

[73] A.K. Rao, S. Srinivas, Buckling of thick rectangular plates., AIAA Journal. 7 (1969) 1645-1646. doi:10.2514/3.5463.

[74] B. Uymaz, M. Aydogdu, Three dimensional mechanical buckling of FG plates with general boundary conditions, Composite Structures. 96 (2013) 174-193. doi:10.1016/j.compstruct.2012.07.033.

[75] G. Jin, Z. Su, S. Shi, T. Ye, S. Gao, Three-dimensional exact solution for the free vibration of arbitrarily thick functionally graded rectangular plates with general boundary conditions, Composite Structures. 108 (2014) 565-577. doi:10.1016/j.compstruct.2013.09.051.

[76] B. Uymaz, M. Aydogdu, Three-Dimensional Vibration Analyses of Functionally Graded Plates under Various Boundary Conditions, Journal of Reinforced Plastics and Composites. 26 (2007) 18471863. doi:10.1177/0731684407081351.

[77] B. Uymaz, M. Aydogdu, Three dimensional shear buckling of FG plates with various boundary conditions, Composite Structures. 96 (2013) 670-682. doi:10.1016/j.compstruct.2012.08.031.

[78] Sh. Hosseini-Hashemi, M. Fadaee, S.R. Atashipour, A new exact analytical approach for free vibration of ReissnerMindlin functionally graded rectangular plates, International Journal of Mechanical Sciences. 53 (2011) 11-22. doi:10.1016/j.ijmecsci.2010.10.002.

[79] H. Nguyen-Xuan, L.V. Tran, C.H. Thai, T. Nguyen-Thoi, Analysis of functionally graded plates by an efficient finite element method with node-based strain smoothing, Thin-Walled Structures. 54 (2012) 1-18. doi:10.1016/j.tws.2012.01.013.
[80] H. Nguyen-Xuan, L.V. Tran, T. Nguyen-Thoi, H.C. Vu-Do, Analysis of functionally graded plates using an edge-based smoothed finite element method, Composite Structures. 93 (2011) 30193039. doi:10.1016/j.compstruct.2011.04.028.

[81] Sh. Hosseini-Hashemi, H. Rokni Damavandi Taher, H. Akhavan, M. Omidi, Free vibration of functionally graded rectangular plates using first-order shear deformation plate theory, Applied Mathematical Modelling. 34 (2010) 1276-1291. doi:10.1016/j.apm.2009.08.008.

[82] X. Zhao, Y.Y. Lee, K.M. Liew, Free vibration analysis of functionally graded plates using the element-free kp-Ritz method, Journal of Sound and Vibration. 319 (2009) 918-939. doi:10.1016/j.jsv.2008.06.025.

[83] L.V. Tran, A.J.M. Ferreira, H. Nguyen-Xuan, Isogeometric analysis of functionally graded plates using higher-order shear deformation theory, Composites Part B: Engineering. 51 (2013) 368-383. doi:10.1016/j.compositesb.2013.02.045.

[84] A. Benachour, H.D. Tahar, H.A. Atmane, A. Tounsi, M.S. Ahmed, A four variable refined plate theory for free vibrations of functionally graded plates with arbitrary gradient, Composites Part B: Engineering. 42 (2011) 1386-1394. doi:10.1016/j.compositesb.2011.05.032.

[85] H.-T. Thai, S.-E. Kim, Closed-form solution for buckling analysis of thick functionally graded plates on elastic foundation, International Journal of Mechanical Sciences. 75 (2013) 34-44. doi:10.1016/j.ijmecsci.2013.06.007.

[86] H.-T. Thai, D.-H. Choi, A refined shear deformation theory for free vibration of functionally graded plates on elastic foundation, Composites Part B: Engineering. 43 (2012) 2335-2347. doi:10.1016/j.compositesb.2011.11.062.

[87] Jeeoot Singh and KK Shukla, Nonlinear flexural analysis of laminated composite plates using RBF based meshless method, Composite Structures.94 (5) (2012), 1714-1720. 